\title{
Hierarchical Logistic Regression Model for Multilevel Analysis: An Application on Use of Contraceptives Among Women in Reproductive Age in Kenya
}

\author{
Linda Vugutsa Luvai, Fred Ongango \\ Department of Applied Statistics and Actuarial Science, Maseno University, Kisumu, Kenya \\ Email address: \\ lindaluvai@gmail.com (L.V. Luvai),fonyango@maseno.ac.ke(F. Ongango) \\ To cite this article: \\ Linda Vugutsa Luvai, Fred Onyango: Hierarchical Logistic Regression Model for Multilevel Analysis: An Application on Use of \\ Contraceptives Among Women in Reproductive Age in Kenya. International Journal of Data Science and Analysis. \\ Vol. 4, No. 5, 2018, pp. 58-78. doi: 10.11648/j.ijdsa.20180405.11
}

Received: July 19, 2018; Accepted: October 8, 2018; Published: November 16, 2018

\begin{abstract}
Contraception allows women and couples to have the number of children they want, when they want them. This is everybody's right according to the United Nations Declaration of Human Rights. Use of Contraceptive also reduces the need for abortion by preventing unwanted pregnancies. It therefore reduces cases of unsafe abortion, one of the leading causes of maternal death worldwide. According to Mohammed, in 2012 an estimated 464,000 induced abortions occurred in Kenya. This translates into an abortion rate of 48 per 1,000 women aged 15-49, and an abortion ratio of 30 per 100 live births. About 120,000 women received care for complications of induced abortion in health facilities. About half (49\%) of all pregnancies in Kenya were unintended and $41 \%$ of unintended pregnancies ended in an abortion. The use of contraceptives in Kenya still remains a big challenge despite the presence of family planning programs through the government and other stake holders. In 2014 a household based cross-sectional study was conducted by Kenya National Bureau of Statistics on women of reproductive age to determine the country's Contraceptive Prevalence Rate and Total Fertility Rate. This dataset is used to exemplify all aspects of working with multilevel logistic regression models, comparison between different estimates and investigation of the selected determinants of contraceptive usage using statistical software, since large surveys in demography and sociology often follow a hierarchical data structure. The appropriate approach to analyzing such survey data is therefore based on nested sources of variability which come from different levels of the hierarchy. When the variance of the residual errors is correlated between individual observations as a result of these nested structures, traditional logistic regression is inappropriate. These analysis showed that different regions have different effects that affect their contraception prevalence. The study also clearly revealed how single level modeling overestimates or underestimates the parameters in study and also helped to bring to understanding of the structure of required multilevel data and estimation of the model via the statistical package $\mathrm{R}$ 3.4.1.
\end{abstract}

Keywords: Multilevel, Contraceptive, Random Intercept, Random Slope

\section{Introduction}

\subsection{Background of the Study}

The basis for the use of contraceptives is the desire to have spaced and limited births by individuals. Unwanted pregnancies and safe abortion occur among women who have limited access to family planning. [10]. Contraception has been identified as an effective means of combating the problem of unwanted pregnancy and unsafe abortion. It is also an effective means of family planning and fertility control and therefore very important in promoting and improving maternal and child health.

"Effective contraception is healthy and socially beneficial to mothers and their children and households." [21] Accessible family planning is essential if women are to enjoy their sexual and reproductive rights in order to get children by choice not by chance. "Mothers who have unintended births tend to suffer postpartum depression, feelings of powerlessness, increased time pressure and a general 
physical health deterioration. They also have poor quality relationships with their children, as they spend less leisure time with them." [21]

Family planning is highly beneficial to women's overall health, particularly in developing countries. Yet, in much of Africa, contraceptive prevalence remains low and the unmet need for family planning remains high. It is hypothesized that the poor quality of family planning service provision in many low income settings is a barrier to contraceptive use [23]. In addition, available evidence shows that most pregnancies in sub-Saharan Africa are unintended or mistimed and the use of family planning methods among this group (reproductive age) remains low. [4]. The standard newspaper on May 27th 2015 stated:" Mistimed and unwanted pregnancies remain common among young women in Kenya several decades after the introduction of modern contraceptive methods.

Contraceptive use among young women remains low compared to older women. Some known barriers to the low uptake include side effects, access to commodities and partner approval. Using evidence from a qualitative study on barriers to modern contraceptive methods uptake among young women in Kenya conducted by Rhoune Ochako and other researchers, it was reported that use of modern contraceptives is surrounded by confusion on appropriate usage and beliefs and myths most of which were hearsay from social networks." [22]

A wider use of contraceptives will lead to fertility decline at all levels and groups of people in Kenya. Family planning workers ought to continue to meet the needs of existing family planning users, and also to address unmet need for family planning since individual tastes, interests, behaviors, etc. differ from one unit to another within each (individual or regional) level, owing to variability among various demographic and geographical factors such as religion, income, place of residence, education, wealth index, age and number of living children one has, and so on. In order for their efforts and approaches to seem to be equally effective, evenly served or acknowledged in some areas they have to come up with programs that are effective and vary considerably. It was therefore necessary to assess the withinand between level variation, and to estimate the true effect of the above-mentioned factors on CPR, in order to implement more effective future family planning policies that target particular units. Where the units at lower level (level-1) are individuals (women aged 15-49) who are nested within units at higher level (level-2) which are regions in the hierarchy.

\subsection{Statement of the Problem}

In the past, a reproductive revolution has swept through much of the developing world, leading to large fertility declines in Asia, Latin America and North Africa. In contrast, fertility declines in Sub-Saharan Africa have been small on average, and the continent's total fertility has continued to be high. Because of this, its population has more than quadrupled between 1950 and 2010 and is expected to double again by 2050 . These demographic trends in SubSaharan Africa have raised concerns about their potential adverse impact on health, social and economic development and the environment. Studies on contraceptive use in Kenya in the past have used single level regression analysis to determine the significance of factors which predict uptake and non-use of contraceptives by women. however, there is need to consider the variations due to hierarchy structure in the data and to allow the simultaneous examination of the effects of group and individual level variables on individual level outcomes while accounting for the non-independence of observations within groups.

\subsection{Objectives}

\subsubsection{General Objective}

The main objective of this work was to build a Hierarchical Logistic Model for Multilevel Analysis on the use of contraceptives among women in the reproductive age in Kenya.

\subsubsection{Specific Objectives}

1. To highlight the importance of multilevel analysis using logistic regression models for studying contraceptive prevalence in Kenya.

2. To determine the true effect of the factors on the contraceptive prevalence taking into consideration the effect of the levels.

3. To investigate the variation of contraceptive between the predictor variables across the regions.

\subsection{Significance of the Study}

The difference in geographical factors, education background, religion, wealth index and age may make a woman to prefer a certain contraceptive over the other. This ought to be achieved with minimum cost and high precision. The model developed from this study will appropriately analyze contraceptive use based on nested sources of variability. The units at lower level (level-1) are nested within units at higher level (level-2). These study will be beneficial to the government in terms of policy making and also it will enrich exiting literature on MLR application. It will also help in establishing conditions necessary for greater uptake of contraception in the country in different regions.

\section{Literature Review}

Studies about contraption have attracted quite a number of researchers in seeking to understand different aspects of contraceptive usage, prevalence, trends among others for example;

A study by Manlove on relationship characteristics and contraceptive use among young adults used bivariate analysis, multivariate logistic and multinomial logistic regressions to assess associations between relationship characteristics and contraceptive use at last sex. From this study it was found that from the multivariate logistic and multinomial logistic regression models that were run to examine associations between relationship characteristics and contraceptive use, while controlling for individual and family 
background factors, relationship characteristics were weakly to only moderately correlated; coefficients ranged from -0.13 (between the intimacy and conflict scales) to 0.39 (between presexual relationship length and overall relationship length). [13].

In another study by Ojokaa a multivariate analysis to determine the significance of various factors affecting contraceptive use, the study on the Patterns and Determinants of Fertility Transition in Kenya Analysis showed that motivation for fertility control and proximity to family planning services were significant factors in determining the contraceptive prevalence. Results from this study showed the need for further research in three areas. The first is a contextual research on the levels, trends and determinants of contraceptive use and fertility in Kenya especially Mount Kenya Region. To conclude the discussion on the determinants of contraceptive use, it can be said that motivation for fertility control, measured here by the number of additional children desired, has a positive and significant effect on using a contraceptive method. The same cannot be said about access to health facilities, save that a related aspect, exposure to messages about family planning is significant and positive. The 2nd [17].

In yet another study, Henry, Juliet, Hassard and Fredrick in Uganda were seeking to understand the contraception knowledge, attitude, perception and sexual behavior among female students in the University. The prevalence ratios were obtained via a modified Poisson regression model using a generalized linear model with Poisson as family and a $\log$ link without an offset but including robust standard errors. The study concluded that knowledge, perceived acceptability and benefits of contraceptive use were nearly universal, but contraceptive use was suboptimal in that setting. Ever trying to terminate a pregnancy was common and a clear indicator of unintended pregnancies. [16]

In seeking to determine the spatial variation in modern contraceptive use and unmet need for family planning in Kenya, Ettarh and Kyobutungi studied the variations in contraceptive use affected by inequalities in physical access to health facilities. In this survey findings of 2008-2009 Kenya Demographic and Health Survey were used for the analysis and multivariate logistic regression was explored to determine whether the influence of distance to the nearest health facility and health facility density, among other covariates influenced modern contraceptive use and unmet need. The study found that modern contraceptive use was significantly less among women who resided more than 5 $\mathrm{Km}$ away from a health facility as compared to those nearest (5 Km or less). Women from counties with higher health facility density were found to be $53 \%$ more likely to use modern contraceptives compared to those who live in counties with low health facility density. [5]

In a study by Worku in Ethiopia on the trends of modern contraceptive use among young married women based on the 2000, 2005 and 2011 Ethiopian Demographic and Health Surveys, a multivariate decomposition analysis was carried out. He found out that, among young married women, modern contraceptive prevalence increased from 6 in 2000 to $16 \%$ in 2005 and to $36 \%$ in 2011 . The decomposition analysis indicated that 34 of the overall change in modern contraceptive use was due to difference in women's characteristics. Changes in the composition of young women's characteristics according to age, educational status, religion, couple concordance on family size, and fertility preference were the major sources of this increase. Twothirds of the increase in modern contraceptive use was due to difference in coefficients. Most importantly, the increase was due to change in contraceptive use behavior among the rural population (33\%) and among Orthodox Christians (16\%) and Protestants (4\%). Logit-based decomposition analysis technique was used for analysis of factors contributing to the recent changes. [24]

A research by Makau, Waititu and Mung'atu in modelling contraceptive use among women in Kenya using multinomial logit, found out that modern contraceptive method is the most preferred method of contraceptive among women, an indication that more women still embrace safe contraception. Marital status, education level, wealth index, area of residence and the number of children a woman has, highly influences the particular contraceptive method to use. However, religion, access to a health facility and age are not key factors a woman would consider while deciding on the particular contraceptive method to use. Multinomial Logistic Regression parameter estimates were found to be consistent estimators and assume a normal distribution as the sample size increases. [11]

From the studies above it is evident that a hierarchical model which will allow us to fit a regression model to the woman contraceptive usage while accounting for systematic unexplained variation among the regions will be necessary so as to assess the within- and between-level variation, and to estimate the true effect of the factors that affect use of contraceptive prevalence in order to allow the implementation of more effective future family planning policies that target particular units at various levels of the hierarchy.

\section{Methodology}

\subsection{Materials and Methods}

Data

The data that we used in this study came from the Kenya Demographic and Health Survey 2014, a nationwide sample survey conducted by Kenya National Bureau of Statistics where a total of 31079 women aged 15-49 years were interviewed. The data was in a hierarchical structure consisting of 2 levels: Individuals and Regions. Excluding missing cases, the sample of the study consists of 31028 women. The hierarchical/multilevel structure is one in which:

1. The coefficients vary by levels (thus, instead of a model such as

$$
y=\alpha+\beta x+\varepsilon
$$


we have

$$
\mathrm{y}=\alpha_{\mathrm{j}}+\beta_{\mathrm{j}} \mathrm{x}+\varepsilon
$$

where the subscripts $j$ index level 1 ,

2. There is more than one variance component.

3. It is a regression with many predictors, including an indicator variable for each level in the data.

More generally, a multilevel model is a model that is considered to be a regression (a linear or generalized linear model) in which the parameters the regression coefficients are given a probability model.

\subsection{Study Variables}

\subsubsection{Dependent Variable}

The dependent variable is 'CurrentUsage'. All the women in the reproductive age were asked whether they are currently using contraceptives or not. Hence, all pregnant women are considered as the non-users in this study. Thus, if a woman is recently using any method then CurrentUsage is coded as ' 1 ' and if not ' 0 '.

\subsubsection{Independent Variables}

1. Demographic Variables

i. Age of the woman $\left(X_{1}\right)$

ii. Number of children $\left(X_{2}\right)$

2. Social Economic Variables
i. Education attainment $\left(X_{3}\right)$
ii. Place of residence $\left(X_{4}\right)$
iii. Religion $\left(X_{5}\right)$
iv. Number of living children $\left(X_{6}\right)$
v. Wealth index $\left(X_{7}\right)$

\subsection{Multilevel Logistic Regression Model}

\subsubsection{Introduction}

Many kinds of data, including observational data collected in the human and biological sciences, have a hierarchical or clustered structure. The two main important uses of Multilevel Models are:

1. Multi-level models take into account the hierarchical structure usually present in data.

2. They provide a flexible framework for analyzing a variety of different types of response variables and for incorporating covariates at different levels of hierarchical structure.

\subsubsection{Assumptions}

Multilevel models have the same assumptions as other major general linear models (e.g., ANOVA, regression), but some of the assumptions are modified for the hierarchical nature of the design (i.e., nested data).

1. Linearity The assumption of linearity states that there is a rectilinear (straight-line, as opposed to non-linear or U-shaped) relationship between variables. However, the model can be extended to nonlinear relationships.

2. Normality The assumption of normality states that the error terms at every level of the model are normally distributed.
3. Homoscedasticity The assumption of homoscedasticity, also known as homogeneity of variance, assumes equality of population variances.

4. Independence of observations

Independence is an assumption of general linear models, which states that cases are random samples from the population and that scores on the dependent variable are independent of each other. One of the main purposes of multilevel models is to deal with cases where the assumption of independence is violated; multilevel models do, however, assume that:

i. The level 1 and level 2 residuals are uncorrelated

ii. The errors (as measured by the residuals) at the highest level are uncorrelated.

\subsubsection{Why Multilevel}

Here are a number of reasons for using multilevel models:

1. Traditional regression techniques treat the units of analysis as independent observations. The failing to recognize the hierarchy in structures is that, standard errors of regression coefficients will be underestimated, leading to an overstatement of statistical significance. Standard errors for the coefficients of higher-level predictor variables will be the most affected by ignoring grouping.

2. Considers Group effects: In many situations a key research question concerns the extent of grouping in individual outcomes, and the identification of 'outlying' groups.

3. Estimating group effects simultaneously with the effects of group level predictors: In a multilevel (random effects) model, the effects of both types of variable can be estimated. To allow for group effects in a traditional (ordinary least squares) regression model we include dummy variables for groups. Such models are called an analysis of variance or fixed effects model. In many cases there will be predictors defined at the group level. In a fixed effects model, the effects of group-level predictors are confounded with the effects of the group dummies, i.e. it is not possible to separate out effects due to observed and unobserved group characteristics.

4. Inference to a population of groups: In a multilevel model the groups in the sample are treated as a random sample from a population of groups. However, while using a fixed effects model, inferences cannot be made beyond the groups in the sample.

\subsection{Multilevel Structures}

The Multilevel structures can be presented in three different ways: A regression which includes indicators for groups is called a varying-intercept model because it can be interpreted as a model with a different intercept within each group. Where $\mathrm{x}$ is the predictor and indicators for $j$ groups. [6] First we consider the model in which the regressions have the same slope in each of the county, and only the intercepts vary. 
The notation $i$ for individuals and $j$ for the region $j$.

\subsubsection{Random Intercept Model}

1. Varying-intercept model:

$$
\gamma_{i j=\alpha+\beta_{1 x_{i j}}}+\mu_{j}+\varepsilon_{i j}
$$

where

$$
\varepsilon \sim N\left(0, \sigma_{e}^{2}\right)
$$

and

$$
\mu \sim N\left(0, \sigma_{\mu}^{2}\right)
$$

The random intercept model has two parts. It's got a fixed part (which is the intercept and the coefficient of the explanatory variable times the explanatory variable) and it's got a random part, so that's this $u_{j}+\varepsilon_{i j}$ at the end. The parameters that we estimate for the fixed part are the coefficients $\alpha, \beta_{1}$ and so on and the parameters that we estimate for the random part are the variances, $\sigma_{u}{ }^{2}$ and $\sigma_{e}{ }^{2}$. The random part is random in the same way that the error term of the single level regression model is random. This means is that the $u_{j}$ and the $\varepsilon_{i j}$ are allowed to vary.

Random Intercept Logit Model

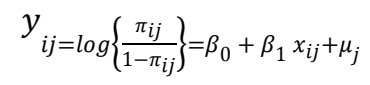

1. $\mathrm{u}_{\mathrm{j}} \sim \mathrm{N}\left(0, \sigma_{\mathrm{u}}^{2}\right)$

2. $\mathrm{x}_{\mathrm{ij}}$ is the observation of $\mathrm{i}^{\text {th }}$ individual in the $\mathrm{j}^{\text {th }}$ region

3. $\pi_{\mathrm{ij}}$ is the response probability i.e.

$$
\pi_{i j=\frac{\exp \left(\beta_{0}+\beta_{1} x_{i j}+\mu_{j}\right)}{1+\exp \left(\beta_{0}+\beta_{1} x_{i j}+\mu_{j}\right.}}
$$

Interpretation of $\beta_{0}$ and $\beta_{1}$ is given by; $\beta_{0}$ interpreted as the log-odds that $y=1$ when $x=0$ and $u=0$ and is referred to as the overall intercept in the linear relationship between the log-odds and $\mathrm{x}$. If we take the exponential of $\beta_{0}, \exp \left(\beta_{0}\right)$, we obtain the odds that $y=1$ for $x=0$ and $u=0$. Compared to the single-level model, where $\beta_{1}$ is the effect of a 1 -unit change in $x$ on the log-odds that $y=1$, in this model it is the effect of $x$ after adjusting for (or holding constant) the group effect $u$. If we are holding u constant, then we are looking at the effect of $x$ for individuals within the same group so $\beta_{1}$ is usually referred to as a cluster-specific effect. In analyzing multilevel data, we are often interested in the amount of variation that can be attributed to the different levels in the data structure and the extent to which variation at a given level can be explained by explanatory variables. On the other hand, $u_{j}$ is the group random effect or level 2 residual and the variance of the intercepts across groups is $\operatorname{var}\left(u_{j}\right)=\sigma_{u}^{2}$ var $\left(u_{j}\right)=\sigma_{u}^{2}$ which is the between group variance adjusted for $x$.

Hypothesis Testing For Random Intercept

In this test we want to know the size of the fixed effects and the amount of variance at each level. We also want to know whether the fixed effects are significant and whether there's a significant amount of variance at level 2.

For the fixed part, hypothesis testing is just the same as for a single level model. We just divide the coefficient by its standard error, to get $\mathrm{Z}$, and then we take the modulus of $\mathrm{Z}$, and if that's bigger than 1.96 (or informally we can use 2) then $\beta_{1}$ is significant at the $5 \%$ level.

$$
\mathrm{Z}=\frac{\beta_{1}}{s . e \beta_{1}}
$$

For the random part we have to fit the model with and without $u_{j}$ and do a likelihood ratio test comparing those 2 models, to see whether $\sigma_{\mathrm{u}}^{2}$ is significant.

\subsubsection{Random Slope Model}

1. Varying-slope model:

$$
y_{i j}=\alpha+\beta_{1} X 1_{i j}+u_{i j} X j+u_{j}+\varepsilon_{j} .
$$

Unlike a random intercept model, a random slope model allows each group line to have a different slope and that means that the random slope model allows the explanatory variable to have a different effect for each group. It allows the relationship between the explanatory variable and the response to be different for each group. This is achieved in the model by adding a random term to the coefficient of $\mathrm{X}$ so that it can be different for each group. The random slope model will therefore have $u_{1} X_{1}$, where $u_{1}$ is different for every group, so that means that this coefficient is different for every group and hence the relationship between $X_{1}$ and $y$ is different for every group.

Hypothesis Testing for Varying slope Model

Fixed part $\beta_{k}$ is significant at the $5 \%$ level if $\mathrm{k} Z_{k} \mathrm{k}>1.96$

Random part: We use a likelihood ratio test:

1. To fit the model with $u_{1 j} X_{1 i j \ldots . . .(1)}$

2. Without $u_{1 j} X_{1 i j \ldots \ldots . . .(0)}$

In other words we are comparing the random slope model to a random intercept model.

The test statistic is again 2 (log (likelihood (1))- $\log$ (likelihood (0))) with 2 degrees of freedom because there are 2 extra parameters in 1 compared to 0 . So we compare the test statistic against the $x_{2}^{2}$ distribution. The null hypothesis is that $\sigma_{u 1}^{2}$ and $\sigma_{u 01}$ are both 0 and hence that a random intercept model is more appropriate than a random slope model.

\subsubsection{Random Intercept, Random Slope Model}

This is where more than one regression coefficient is allowed to vary by group. The intercepts and the slopes are treated as observations from a bivariate normal distribution.

1. Varying-intercept, varying-slope model:

$$
\begin{gathered}
Y i j=(b 0 j+\beta 0)+(b 1 j+\beta 1) X i j+\varepsilon i j \\
b_{0 j} \sim N\left(0, \tau_{1}^{2}\right) ; \\
b_{1 j} \sim N\left(0, \tau_{2}^{2}\right) ; \\
\operatorname{cov}(\mathrm{b} 0 \mathrm{j}, \mathrm{b} 1 \mathrm{j})=\tau 12 .
\end{gathered}
$$

The varying slopes are interactions between the predictor $\mathrm{X}$ and the group indicators. 


\subsection{Parameter Estimation}

Iterative Generalized Least Square Methods is a good estimate of parameters in a multilevel model. first we consider having known the variance in a 2-level model we could construct a block diagonal matrix V, but know we can use the General Least Square method to obtain the estimators of fixed coefficient namely;

$$
\begin{gathered}
\widehat{\beta}=(\mathrm{XtV}-1 \mathrm{X})-1 \mathrm{XtV}-1 \mathrm{Y} \\
\mathrm{ij}=\beta 0+\beta 1 \mathrm{xij}+\mu 0 \mathrm{j}+\varepsilon 0 \mathrm{ij} \\
\operatorname{Var}\left(\varepsilon_{0 i j}\right)=\sigma_{e 0}^{2} \\
\operatorname{Var}\left(\mu_{0 j}\right)=\sigma_{\mu 0}^{2}
\end{gathered}
$$

where in this case

$$
\mathbf{X}=\left[\begin{array}{ccc}
1 & \cdots & x_{11} \\
\vdots & \ddots & \vdots \\
1 & \cdots & x_{n m}
\end{array}\right] \mathbf{Y}=\left[\begin{array}{c}
y_{11} \\
\vdots \\
y_{1 m}
\end{array}\right] \quad \mathbf{V}=\left[\begin{array}{ccc}
1 & \cdots & v_{11} \\
\vdots & \ddots & \vdots \\
1 & \cdots & v_{1 m}
\end{array}\right]
$$

where we have m level 2 units and $n_{j}$ level 1 units in the $j^{\text {th }}$, level 2 units.

Logit Model Estimation

$$
\int \mathrm{f}_{\mathrm{y}} \mathrm{y}(\mathrm{obs}) \partial \beta=\frac{\left.\left|\omega^{\prime} 1 / 2\right| \mathcal{L}_{\theta}\right|^{-1}}{2 \pi \sigma^{2 n / 2}} \exp \left\{\frac{-r^{2}(\theta)}{2 \sigma^{2}}\right\} \int \exp \left\{\frac{-\left\|\mathcal{R}_{X}\left(\beta-\beta_{0}\right)\right\|^{2}}{2 \sigma^{2}}\right\} d \beta
$$

which can be evaluated with the change of variables,

$$
v=R_{X}\left(\beta-\hat{\beta_{\theta}}\right)
$$

The Jacobian determinant of the transformation from $\beta$ to $\mathrm{v}$ is $\left|R_{X}\right|$. Therefore we are able to write the integral as,

$$
\int \mathrm{f}_{\mathrm{y}} \mathrm{y}(\mathrm{obs}) \partial \beta=\frac{\left.\left|\omega^{\prime} 1 / 2\right| \mathcal{L}_{\theta}\right|^{-1}}{2 \pi \sigma^{2 n / 2}} \exp \left\{\frac{-r^{2}(\theta)}{2 \sigma^{2}}\right\} \int \exp \left\{\frac{-\|v\|^{2}}{2 \sigma^{2}}\right\}\left|\mathcal{R}_{X}\right| d \nu
$$

which simplifies to,

$$
\int_{\mathrm{f}_{\mathrm{y}} \mathrm{y}}(o b s) \partial \beta=\frac{\left.\left|\omega^{\prime} 1 / 2\right| \mathcal{L}_{\theta}\right|^{-1}\left|\mathcal{R}_{x}\right|^{-1}}{2 \pi \sigma^{2(n-p) / 2}} \exp \left\{\frac{-r^{2}(\theta)}{2 \sigma^{2}}\right\}
$$

Minus twice the log of this integral is the (unprofiled) REML criterion,

$$
-2 \mathcal{L}_{R}\left(\theta,\left.\sigma^{2}\right|_{\text {yobs }}\right)=\frac{\log \left|\mathcal{L}_{\theta}\right|^{2}\left|\mathcal{R}_{X}\right|^{2}}{W}+(n-p) \log \left(2 \pi \sigma^{2}\right)+\frac{r_{\theta}^{2}}{\sigma^{2}}
$$

We note that because $\beta$ gets integrated out, the REML criterion cannot be used to find a point estimate of $\beta$. However, we follow others in using the maximum likelihood estimate, $\hat{\beta_{\theta}}$, at the optimum value of $\theta=\theta$. The REML estimate for $\sigma^{2}$ is,

$$
\hat{\sigma}_{\theta}^{2}=\frac{r^{2}(\theta)}{n-p}
$$

which leads to profiled REML criterion,

$$
-2 \mathcal{L}_{R}\left(\theta, \sigma^{2} \mid y o b s\right)=\frac{\left.\log \left|\mathcal{L}_{\theta}\right|^{2} R_{X}\right|^{2}}{W}+(n-p)\left\{1+\log \left(\frac{2 \pi r^{2}(\theta)}{n-p}\right)\right\}
$$

\section{Analysis, Results and Discussions}

In order to use the $\mathrm{R}$ package for multilevel analysis, we organized the data to reflect the data's hierarchical structure in the analysis. The KDHS data was therefore first sorted in such a way that all records for the same highest level (level2: Regions) were grouped together. The selected covariates used in this study found were all found to be significant in the analysis which was done before to start multilevel analysis. The multilevel modeling process for this hierarchical data was therefore done step by step. The first step being to 
examined the null model of overall probability of contraceptive use without adjustment for predictors. This was followed by the second step which included the analysis of (both single and multilevel analysis model, and then random slope multilevel analysis for each of the selected explanatory variables. Third step considered building a model for multilevel logistic regression analysis and that of single level analysis. Finally, the likelihood ratio test was used to determine significance of each model as a whole as well as to determine significance of the individual coefficients.

\subsection{Descriptive Analysis}

Contraceptive usage by women who were sampled is as shown below:

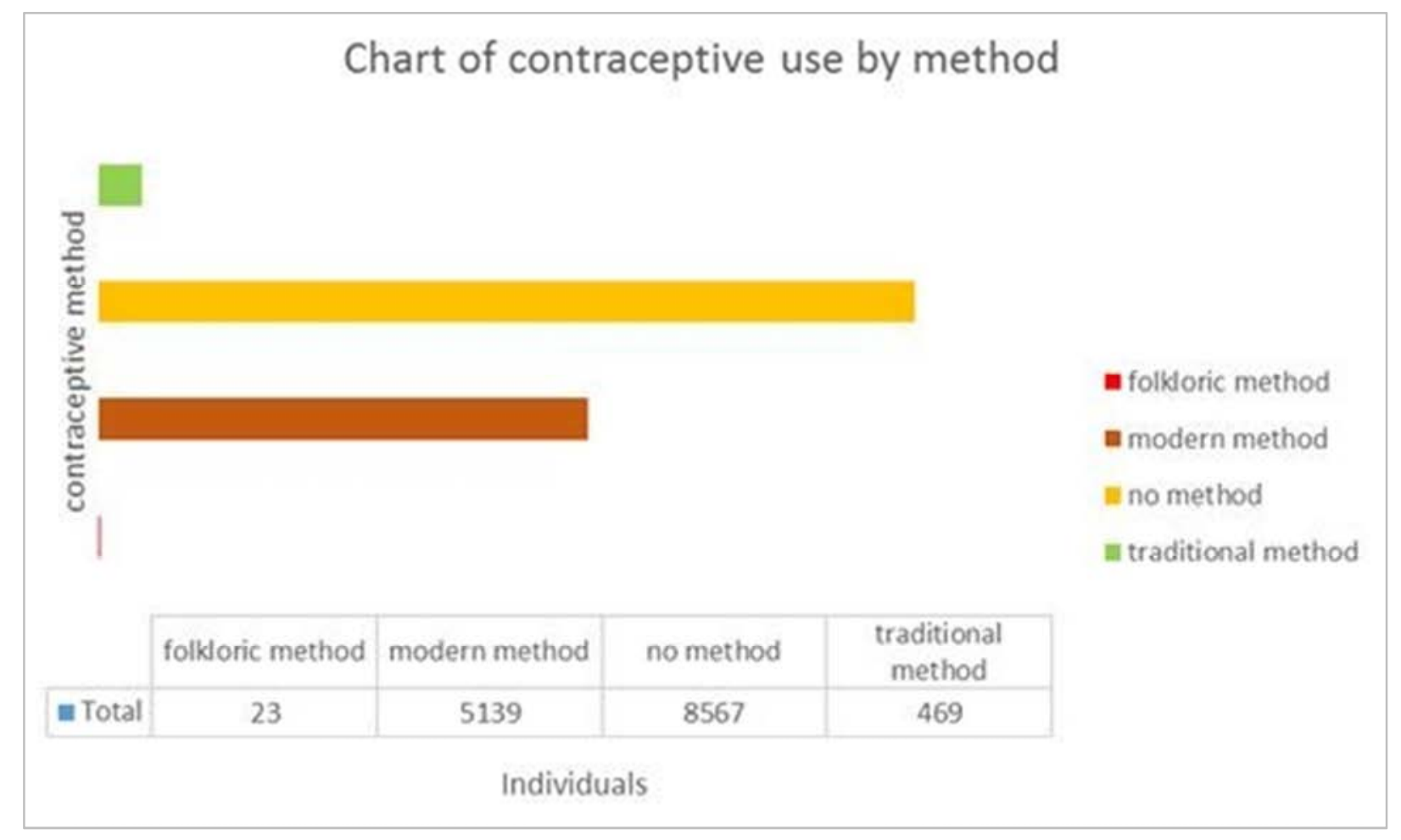

Figure 1. Current Usage of Contraceptive by Method.

A greater percentage $(61 \%)$ of the women in the reproductive age in Kenya have not been using any contraception method. However $(35.475 \%)$ have embraced the modern method while folkloric method $(0.164 \%)$ is the least embraced method. Among those using contraception $91.63 \%$ prefer modern method while $7.94 \%$ were reported to use traditional method and the least common method was folkloric method where only $0.42 \%$ use it.

\subsection{Intercept Only Model}

\subsubsection{Null Model}

The null or empty two-level model that is a model with only an intercept and Regional effects.

$$
\log \left\{\frac{\pi_{i j}}{1-\pi_{i j}}\right\}=\beta_{0}+\mu_{0 j}
$$

The intercept $\beta_{0}$ is shared by all regions while the random effect $\mu_{0 j}$ is specific to region $\mathrm{j}$. The random effect was assumed to follow a normal distribution with variance $\sigma_{u 0}^{2}$. From the model estimates (using Laplacian Approximation), we saw that the logs-odd of using contraception in the region is estimated as
Table 1. Null model.

\begin{tabular}{ll}
\hline & Model 1 \\
\hline (Intercept) & $-0.72^{*}$ \\
& $(0.36)$ \\
AIC & 39806.68 \\
BIC & 39823.37 \\
Log Likelihood & -19901.34 \\
Num. obs. & 31038 \\
Num. groups: Region & 8 \\
Var: Region (Intercept) & 1.17 \\
\hline
\end{tabular}

$* * * \mathrm{p}<0.001, * * \mathrm{p}<0.01, * \mathrm{p}<0.05$

$\beta_{0}=-0.7207$. This means that the odds of using contraception in an average region is $\exp (-0.7207)=0.4864$ and the corresponding probability will be $\frac{0.4864}{1+0.4864}=0.3272$. The intercept for region $j$ is $-0.7207+\mu_{o j}$, where the variance of $\mu_{o j}$ was estimated as $\sigma_{u 0}^{2}=1.174$. There was a strong evidence that between regions variance is non zero. The ICC1 value of 0.04911 from the null model indicates that $5 \%$ of the variation in contraceptive usage can be explained at the regional level. The ICC2 value of 0.9950 indicates that Regions can be very reliably differentiated in terms of Contraception Usage. The two regions with the lowest 
probability of using contraception i.e (largest negative values of $\mu^{j} u^{j}$ ) are North Eastern and Eastern Regions, while Central and Western Regions have the highest response probability (largest positive values of $\mu^{j} u^{j}$ ) as shown in the figures below.

The plot shows the estimated residuals for all 8 regions in the sample. For a substantial number of regions, the $95 \%$ confidence interval does not overlap the horizontal line at zero, indicating that usage of contraception.

\section{Random effects of (Intercept)}

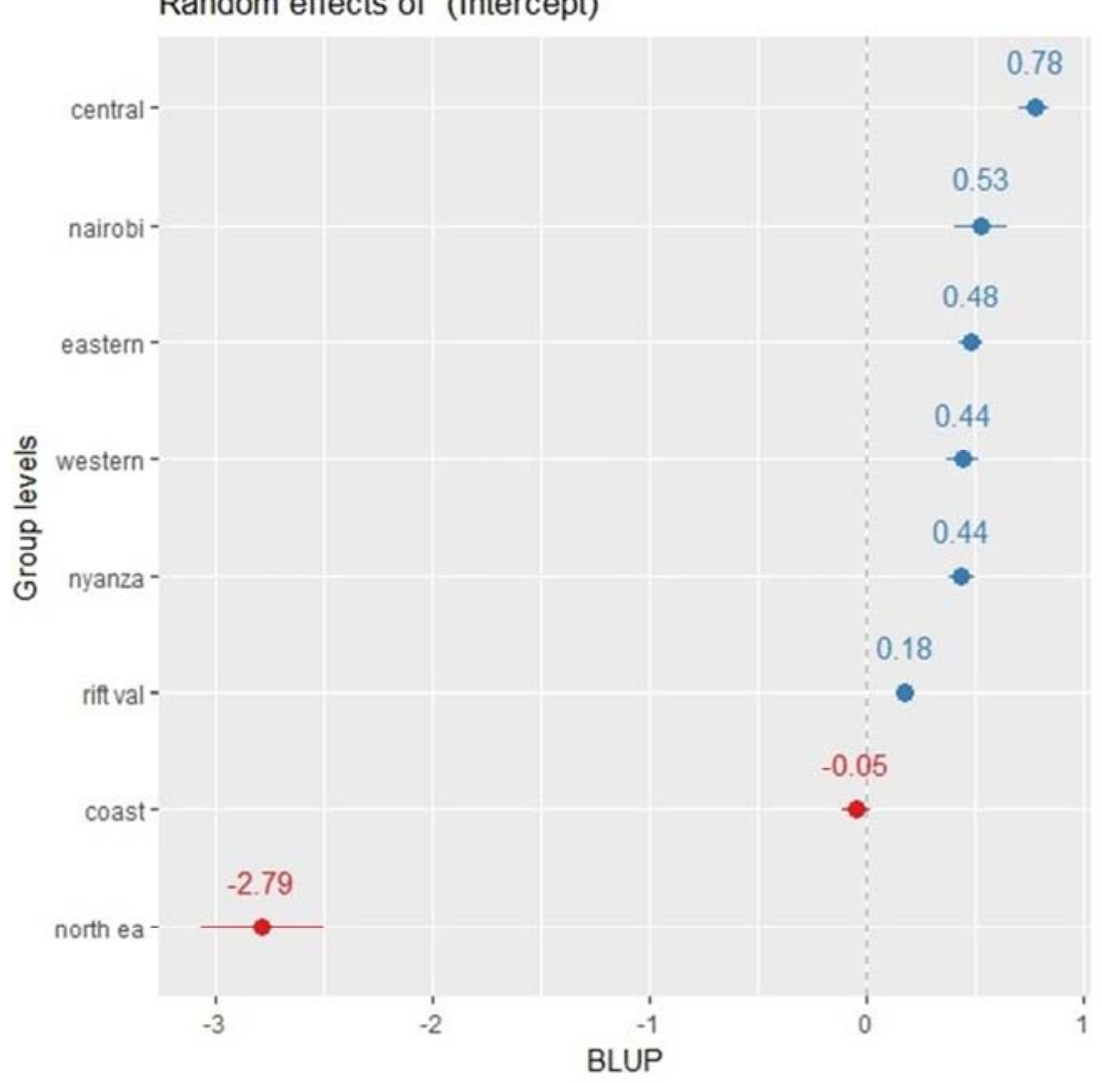

a)

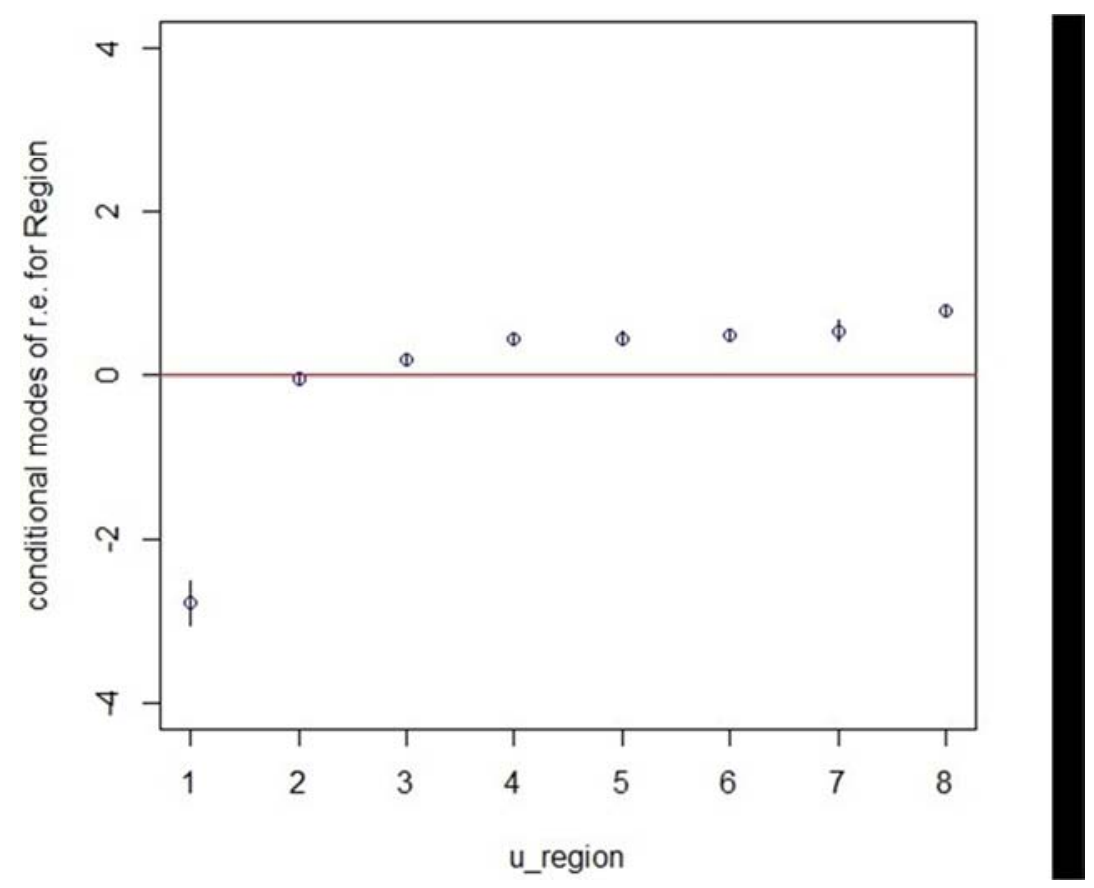

b)

Figure 2. a) Random Effects of Regions On Usage of Contraception; b) Residual Plot for Regions. 
method (s) in these regions is significantly above average (above the zero line).

\subsubsection{Random Intercept with Explanatory Variables}

The estimation procedure used by $\mathrm{R}$ optimizes a function of the log likelihood using penalized iteratively re-weighted least squares.

$$
\log \left\{\frac{\pi_{i j}}{1-\pi_{i j}}\right\}=\beta_{0}+\beta_{1} X_{1}+\mu_{0 j}
$$

For a woman aged 20, the log-odds of using contraception ranges from about -2.4 to 2.4 depending on which region she lives in. This translates to a range in probabilities of $\frac{\exp (-2.4)}{1+\exp (-2.4)}=0.08$ to $\frac{\exp (2.4)}{1+\exp (2.4)}=0.9168$

Likewise for a woman with 5 children, the log-odds of using contraception ranges from -0.7 to 0.7 depending on the region she lives in. This can be translated to be

$$
\frac{\exp (-0.7)}{1+\exp (-0.7)}=0.3318 \text { to } \frac{\exp (0.7)}{1+\exp (0.7)}=0.6681
$$

There are strong regional effects for both age and NoLC that a woman has.

Table 2. Table of parameters and standard errors of univariate single level logistic model and multilevel model predicting the probability of contraceptive use

\begin{tabular}{|c|c|c|c|}
\hline & Single Level & Multilevel & Over/Underestimation \\
\hline (Intercept) & $\begin{array}{l}-3.24 * * * \\
(0.09)\end{array}$ & $\begin{array}{l}-3.22 * * * \\
(0.24)\end{array}$ & $0.62 \%$ \\
\hline AgeG20-24 & $\begin{array}{l}1.72 * * * \\
(0.06)\end{array}$ & $\begin{array}{l}1.71 * * * \\
(0.06)\end{array}$ & $0.58 \%$ \\
\hline AgeG25-29 & $\begin{array}{l}2.05^{* * *} \\
(0.06)\end{array}$ & $\begin{array}{l}2.00^{* * *} \\
(0.06)\end{array}$ & $2.5 \%$ \\
\hline AgeG30-34 & $\begin{array}{l}1.94^{* * *} \\
(0.06)\end{array}$ & $\begin{array}{l}1.87 * * * \\
(0.06)\end{array}$ & $3.74 \%$ \\
\hline AgeG35-39 & $\begin{array}{l}1.69^{* * *} \\
(0.07)\end{array}$ & $\begin{array}{l}1.59 * * * \\
(0.07)\end{array}$ & $6.289 \%$ \\
\hline AgeG40-44 & $\begin{array}{l}1.32^{* * * *} \\
(0.07)\end{array}$ & $\begin{array}{l}1.18^{* * * *} \\
(0.07)\end{array}$ & $11.86 \%$ \\
\hline AgeG45-49 & $\begin{array}{l}0.70^{* * *} \\
(0.08)\end{array}$ & $\begin{array}{l}0.53^{* * * *} \\
(0.08)\end{array}$ & $32 \%$ \\
\hline PORurban & $\begin{array}{l}0.07 * \\
(0.03)\end{array}$ & $\begin{array}{l}0.12 * * * \\
(0.03)\end{array}$ & $41.67 \%$ \\
\hline Religionno relig & $\begin{array}{l}0.64 * * * \\
(0.13)\end{array}$ & $\begin{array}{l}0.44 * * * \\
(0.13)\end{array}$ & $45.45 \%$ \\
\hline Religionother & $\begin{array}{l}0.36 \\
(0.32)\end{array}$ & $\begin{array}{l}0.13 \$ \\
(0.33)\end{array}$ & $176.9 \%$ \\
\hline Religionprotesta & $\begin{array}{l}1.11 * * * \\
(0.06)\end{array}$ & $\begin{array}{l}0.92 * * * \\
(0.06)\end{array}$ & $20.65 \%$ \\
\hline Religionroman ca & $\begin{array}{l}1.09 * * * \\
(0.06)\end{array}$ & $\begin{array}{l}0.86^{* * * *} \\
(0.07)\end{array}$ & $26.74 \%$ \\
\hline WIpoorer & $\begin{array}{l}-0.24 * * * \\
(0.04)\end{array}$ & $\begin{array}{l}-0.24 * * * \\
(0.04)\end{array}$ & \\
\hline WIpoorest & $\begin{array}{l}-0.89 * * * \\
(0.05)\end{array}$ & $\begin{array}{l}-0.87 * * * \\
(0.05)\end{array}$ & $2.3 \%$ \\
\hline WIricher & $\begin{array}{l}0.01 \\
(0.04)\end{array}$ & $\begin{array}{l}0.00 \\
(0.04)\end{array}$ & \\
\hline WIrichest & $\begin{array}{l}0.00 \\
(0.05)\end{array}$ & $\begin{array}{l}-0.02 \\
(0.05)\end{array}$ & \\
\hline NoLC & $\begin{array}{l}0.25^{* * *} \\
(0.01) \$\end{array}$ & $\begin{array}{l}0.28^{* * * *} \\
(0.01)\end{array}$ & $10.71 \%$ \\
\hline Educationno educa & $\begin{array}{l}-1.58 * * * \\
(0.08)\end{array}$ & $\begin{array}{l}-1.50 * * * \\
(0.08)\end{array}$ & $5.33 \%$ \\
\hline Educationprimary & $\begin{array}{l}0.05 \\
(0.05)\end{array}$ & $\begin{array}{l}-0.02 \\
(0.05)\end{array}$ & \\
\hline Educationsecondar & $\begin{array}{l}0.07 \\
(0.05)\end{array}$ & $\begin{array}{l}0.03 \\
(0.05)\end{array}$ & $133.33 \%$ \\
\hline AIC & 33635.49 & 33247.94 & \\
\hline BIC & 33802.35 & 33423.14 & \\
\hline Log Likelihood & $\begin{array}{l}-16797.75 \\
33595.49\end{array}$ & -16602.97 & \\
\hline $\begin{array}{l}\text { Deviance } \\
\text { Var: Region (Intercept) }\end{array}$ & $\begin{array}{l}33595.49 \\
0.39\end{array}$ & & \\
\hline
\end{tabular}
with random intercept only.

$* * * \mathrm{p}<0.001,{ }^{* *} \mathrm{p}<0.01,{ }^{*} \mathrm{p}<0.05$ 
67 Linda Vugutsa Luvai and Fred Ongango: Hierarchical Logistic Regression Model for Multilevel Analysis: An Application on Use of Contraceptives Among Women in Reproductive Age in Kenya

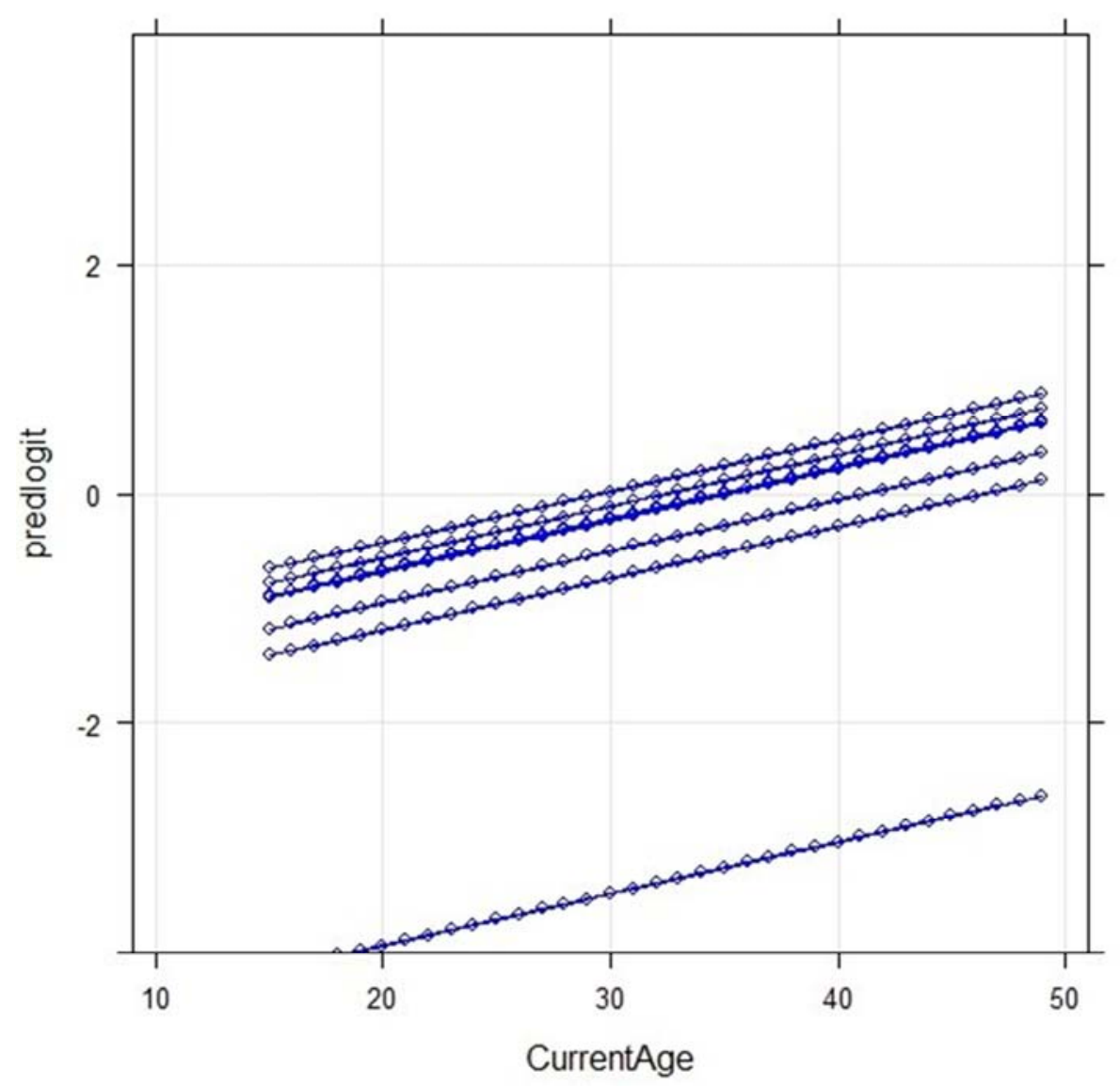

Figure 3. Predicted probabilities against Age.

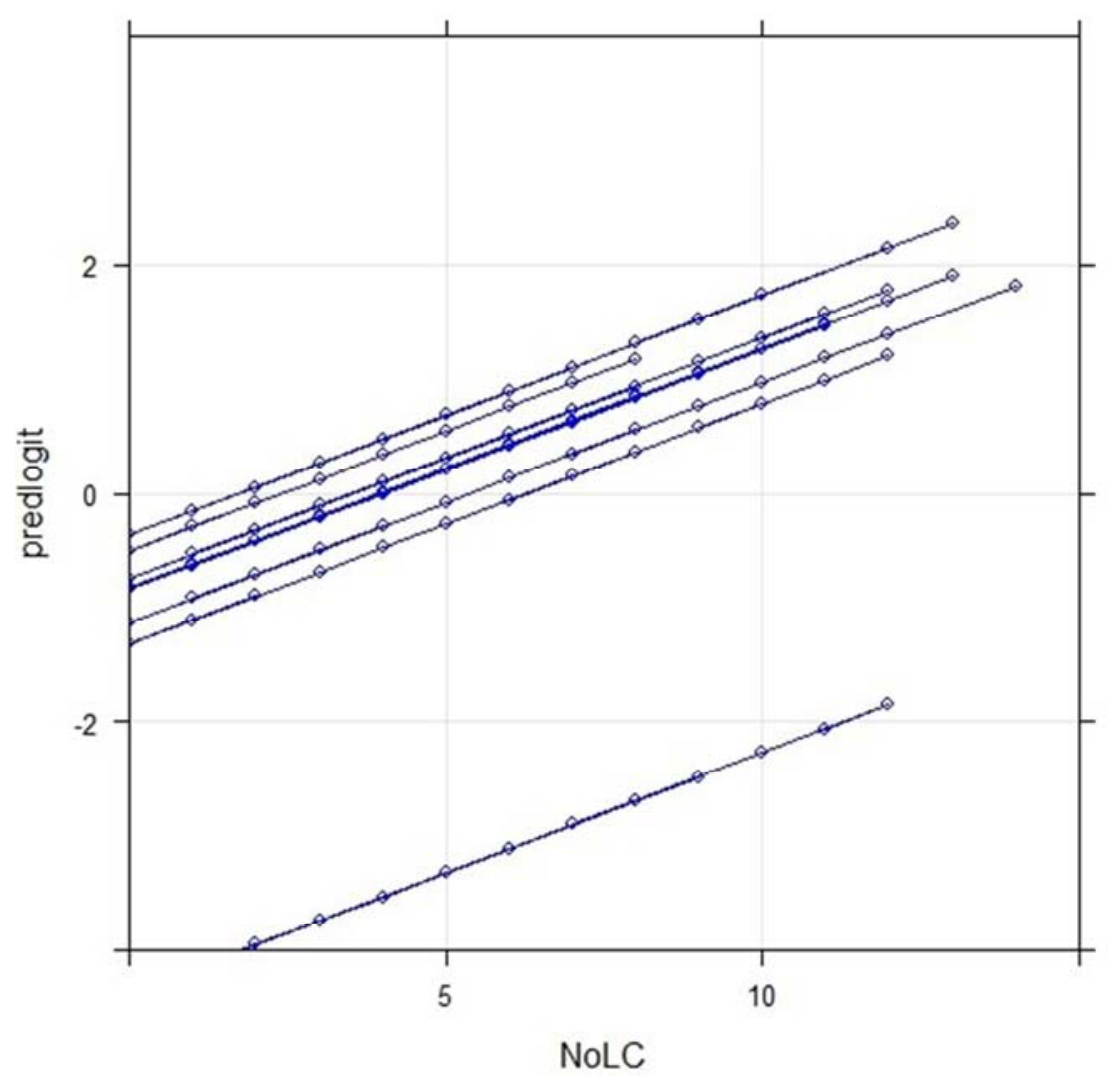

Figure 4. Predicted probabilities against NoLC. 


\subsection{Multilevel Univariate Analysis}

In this univariate analysis represented in Table 2 each of the models presents a random intercept and a fixed slope for the variable.

$$
\beta 0 i j=\beta 0+\mu 0 j+\mu 0 i j
$$

It was observed that there existed a significant differences between the $\beta$ coefficients of the single level and multilevel explanatory variables. The $\beta$ coefficients of the single level model was underestimated in comparison with the multilevel analysis model. The results showed that all the explanatory variables significantly influence contraception usage by a woman at $(\mathrm{p}<0.001)$ For the AgeG 20-24, 25-29, 30-34 the variance across the groups is constant while it varies by $14.29 \%$ of the Age group $35-39,40-44$ and varies by $25 \%$ for AgeG 45 - 49. This shows that some Regions depict a high tendency on the contraceptive usage as age increases while others reveal low less usage as age increases. When the effect of multilevel analysis is not taken into consideration the $\beta$ coefficients for the explanatory variables are overestimated as shown in the last column of table 2. For example the estimates of NoLC are underestimated by $10.71 \%$

\subsection{Multilevel Vs Single Level}

If we compare the two sets of results, the coefficients of the education levels, No Education, primary Education and Secondary Education, increase when the random effect is added. The ratio of the multilevel to single-level estimate is for 0.9589 for noeduaction, 1.3 for primary level and 1.161 for secondary level. In contrast, the coefficient of religion decreases when the region random effect is added. The ratio will not apply here because we have already seen that the mean of the individual woman on religion varies substantially from region to region. Furthermore, we expect that the individual religion is associated with unobserved regional-level determinants of contraception usage, for example the availability of contraception in health service providers. If there are variety and available contraception services are offered in less-deprived areas, and these areas have higher use of contraception from medically-trained providers, we would expect that controlling for unobserved regional characteristics in the multilevel model will reduce the effect of religion. One unit increase in the predictor Number of Living Children (NoLC), corresponds to a 0.332401 increase in the outcome Current Usage. Likewise, the logs-odd of using contraception for a woman living in an urban area is 0.219566 higher than that of a woman living in the rural area. Furthermore, the categorical predictor WI; WIpoorer has a coefficient of -0.228057 ; which means, contraception usage logs-odd of a woman in the group of WIpoorer is 0.228057 lower than the contraception usage of WImiddle class. On the other hand, the logs-odd of contraception usage among the group WIricher is 0.0060181 higher than that of WImiddle class. Likewise the logs-odd of using contraception for the group with no education is 1.7543 less that an individual who has education, while for and individual with primary education is -0.2100 less compared to the one having higher education.

Table 3. Table of Single Level Analysis vs Multilevel.

\begin{tabular}{lllll}
\hline \multirow{2}{*}{ Parameter } & Single level & & Multilevel & \\
\cline { 2 - 5 } & Estimate & Std Error & Estimate & Std Error \\
\hline$\beta_{0}$ (Intercept) & -1.8326 & 0.00888 & -1.685 & 0.238081 \\
$\beta_{1}$ (CurrentAge) & 0.0021 & 0.002 & -0.004 & 0.002 \\
$\beta_{2}$ (NoLC) & 0.303 & 0.0093 & 0.3324 & 0.0097 \\
POR & 0.1796 & 0.0306 & 0.2196 & 0.0312 \\
No Religion & 0.7725 & 0.1249 & 0.5436 & 0.1273 \\
Other Religion & 0.4231 & 0.3192 & 0.1725 & 0.3231 \\
Protestant & 1.1577 & 0.055 & 0.9682 & 0.0622 \\
RomanCatholic & 1.1059 & 0.0591 & 0.8832 & 0.0664 \\
WIPoorer & -0.2277 & 0.0398 & -0.2281 & 0.0401 \\
WIPoorest & -0.864 & 0.0454 & -0.8587 & 0.0462 \\
WIRicher & 0.0694 & 0.0401 & 0.0602 & 0.0404 \\
WIRichest & 0.059 & 0.0454 & 0.029 & 0.0466 \\
noEducation & -1.8295 & 0.0776 & -1.7543 & 0.0785 \\
PrimaryEducation & -0.21 & 0.0484 & -0.2731 & 0.049 \\
Secondary Education & -0.2595 & 0.0477 & -0.3013 & 0.0481 \\
\hline
\end{tabular}

\subsection{Random Slope Models}

\subsubsection{Random Slope for Wealth Across Regions}

$$
Y i j=\beta 0+(\beta 1+\mu 1 j) X 7+\mu 0 j+\varepsilon 0 i j
$$

where;

$\beta_{0}$ is the intercept (the logs odd of using contraception for an individual living in an average region), $\beta_{1}$ is the effect on the log-odds of a category increase in wealth index (the average change in contraceptive usage across all the groups for a change in wealth index),

$\mu 1 j$ and $\mu 0 j$ are the random intercepts,

$\sigma^{2}$ is the residual.

The logs-odd of contraception usage at region $i$ was estimated as -0.44643 and the variance of the slopes among the regions is 0.004674 higher for WIpoorer than for WImiddle, 0.721991 higher for WIpoorest than for 
WImiddle, 0.005265 higher for WIricher than WImiddle and 0.018249 higher for WIrichest as compared to WImiddle. For an average region we predict a decrease of 0.44643 units of

$$
\sigma_{u}^{2}=0.004674, \sigma_{v}^{2}=0.721991 \sigma_{w}=0.005265 \sigma_{x}^{2}=0.018249,
$$

as shown the table (s) below.

The estimated variance for the intercept is 0.798369 which is the variability across the regions with an average WI. Only WIpoorer and WIpoorest was found to be significant. WIpoorer had a logs-odd of -0.17261 lower to that of contraception usage when the wealth index decreases by one unit. The estimated variances are:

Table 4. Table of varying Wealth across regions.

\begin{tabular}{lll}
\hline & WI & WI:POR \\
\hline Intercept) & $-0.45(0.28)$ & $-0.49(0.31)$ \\
WIpoorer & $-0.17^{*}(0.07)$ & $-0.17^{*}(0.08)$ \\
WIpoorest & $-0.94^{* *}(0.29)$ & $-0.99^{* *}(0.30)$ \\
WIricher & $-0.03(0.07)$ & $0.04(0.08)$ \\
WIrichest & $-0.04(0.08)$ & $0.18(0.11)$ \\
PORurban & & $0.09(0.06)$ \\
WIpoorer: PORurban & & $0.10(0.09)$ \\
WIpoorest: PORurban & & $0.37^{* * *}(0.10)$ \\
WIricher: PORurban & & $-0.14(0.08)$ \\
WIrichest: PORurban & & $-0.29^{* *}(0.10)$ \\
\hline
\end{tabular}

${ }^{* *} p<0.001,{ }^{* *} p<0.01,{ }^{*} p<0.05$

The estimated variance for the intercept is 0.798369 which is the variability across the regions with an average WI. Only WIpoorer and WIpoorest was found to be significant. WIpoorer had a logs-odd of -0.17261 lower to that of WImiddle while WIpoorest had a logs-odd of -0.98889
WImiddle while WIpoorest had a logs-odd of -0.98889 lower than that of WImiddle and had a slope of 0.09867 and 0.36594 respectively. This means that contraceptive usage is lower among the group of Poorer and Poorest irrespective of where one resides. This can be seen from the figures below.

Table 5. Random Effects of Varying wealth across Regions

\begin{tabular}{|c|c|c|c|c|c|c|}
\hline Groups Name & Variance & Std. Dev. & Corr & & & \\
\hline Region (Intercept) & 0.798369 & 0.89351 & & & & \\
\hline WIpoorer & 0.010973 & 0.10475 & 0.79 & & & \\
\hline WIpoorest & 0.651199 & 0.80697 & 0.84 & 0.77 & & \\
\hline WIricher & 0.005321 & 0.07294 & -0.70 & -0.86 & -0.94 & \\
\hline WIrichest & 0.028990 & 0.17026 & -0.92 & -0.97 & -0.84 & 0.84 \\
\hline
\end{tabular}

The effect of religion on the log-odds of using contraceptives in a given region $j$ is estimated as 0.6529 for a woman with no religion in relation to a Muslim woman, 0.3350 for a woman who is from other religion, 1.1855 for a protestant woman and 1.0512 for a catholic woman, and the between-region variance in the effect of religion is estimated as 0.1796 for a woman with no religion, 0.9560 for that with other religion, 0.8609 for a protestant woman and 0.5312 for the catholic. Because religion has been centered about its sample mean, the intercept variance $\sigma_{u 0}^{2} \sigma_{u 0}^{2}=0.8136$ which is the between-region variance in the log-odds of contraceptive usage at the mean of the religion. The p-value of a woman from other religion was not significant. Contraception usage of a woman in other religion was not influenced by religion. A woman who is a protestant is highly influenced by her religion as compared to others.

\subsubsection{Varying NoLC with Current Age}

$$
Y_{i j}=\beta_{0}+\beta_{1}+\pi_{1} X_{6}+\pi_{2} X_{1}+\varepsilon_{0 i j}
$$

lower than that of WImiddle and had a slope of 0.09867 and 0.36594 respectively. This means that contraceptive usage is lower among the group of Poorer and Poorest irrespective of where one resides. This can be seen from the figures below. means that POR was not significantly different across the regions. While CurrentAge and NoLC was found to be significant at $(p<0.001)$. Therefore CurrentAge and the NoLC a woman has, influences her contraception usage.

Table 6. Table of Varying No LC with Current Age.

\begin{tabular}{ll}
\hline (Intercept) & $-0.81 * * *(0.07)$ \\
CurrentAge & $0.07 * * *(0.00)$ \\
NoLC & $0.12 * * *(0.01)$ \\
I (CurrentAge $\left.{ }^{2}\right)$ & $-0.00 * * *(0.00)$ \\
PORurban & $0.05 * * *(0.02)$ \\
CurrentAge:NoLC & $-0.00 * * *(0.00)$ \\
Var: Region (Intercept) & 0.03 \\
Var: Region PORurban & 0.00 \\
Cov:Region (Intercept) PORurban & -0.01 \\
Var:Residual & 0.20 \\
\hline
\end{tabular}

$* * * \mathrm{p}<0.01, * * \mathrm{p}<0.01, * \mathrm{p}<0.05$ 
Table 7. Table of varying NoLC across regions.

\begin{tabular}{lllll}
\hline Random effects: Groups Name & Variance & Std. Dev. & Corr & \\
\hline Region (Intercept) & 0.76844 & 0.8766 & & 0.61 \\
NoLC & 0.02323 & 0.1524 & z value & $\operatorname{Pr}(>|\mathbf{z}|)$ \\
Fixed effects: & Estimate & Std. Error & -4.285 & $1.83 e-05 * * *$ \\
(Intercept) & -1.28057 & 0.29884 & 4.733 & $2.22 e-06 * * *$ \\
NoLC & 0.25578 & 0.05405 & \\
\hline
\end{tabular}

${ }^{* * *} p<0.001,{ }^{* *} p<0.01,{ }^{*} p<0.05$

The output of this mixed model suggested that there was a strong positive correlation (Corr; $\mathrm{r}=0.61$ ) between the intercepts and the slopes (NoLC) among Regions. That is, among Regions, intercepts and slopes were found to be completely independent (NoLC a woman has and how it influences her contraceptive usage is different in different Regions). A unit change in the NoLC leads to 0.25578 change in contraceptive usage among women in the reproductive age.

\subsubsection{Varying Current Age}

$$
Y_{i j}=\beta_{0}+\left(\beta_{1}+\mu_{1 j}\right) X_{1}+\mu_{0 j}+\varepsilon_{0 i j}
$$

From the analysis, it was evident that contraception usage logs odd increases by about $54.597 \%$ for each additional year of age while it is highly significant at the quadratic term and also significant at the linear term. The regional intercept 0.8065 and the slope CurrentAge 0.000298. Varying the slope fits the data better than just varying the intercept. When comparing this model with that without the slope, we found out that the slope was significant. This means that CurrentAge in relation to contraceptive usage varies across the regions and that there is an exponential decrease in contraceptive usage as age increases. The intercept and the slope have a negative correlation -0.301 , this means that for regions with higher contraceptive usage among women in reproductive age there tends to be a smaller increase in contraceptive usage.

Table 8. Table of varying Age across regions.

\begin{tabular}{|c|c|c|c|c|}
\hline Random effects: Groups Name & Variance & Std. Dev. & Corr & \\
\hline Region (Intercept) & 0.806481 & 0.89804 & & \\
\hline CurrentAge & 0.000298 & 0.01726 & 0.23 & \\
\hline Fixed effects: & Estimate & Std. Error & $\mathrm{z}$ value & $\operatorname{Pr}(>|z|)$ \\
\hline (Intercept) & -9.1688295 & 0.3553504 & -25.80 & $<2 e-16 * * *$ \\
\hline CurrentAge & 0.5459720 & 0.0123820 & 44.09 & $<2 e-16 * * *$ \\
\hline$I\left(\right.$ CurrentAge $\left.{ }^{2}\right)$ & -0.0080802 & 0.0001647 & -49.06 & $<2 e-16 * * *$ \\
\hline$I\left(C r r n t A^{2}\right)$ & 0.405 & -0.834 & & \\
\hline
\end{tabular}

${ }^{* * *} p<0.001,{ }^{* *} p<0.01,{ }^{*} p<0.05$

\subsubsection{Varying Education}

$$
Y_{i j}=\beta 0+(\beta 1+\mu 1 j) X 3+\mu 0 j+\varepsilon 0 i j
$$

where;

1. $\beta_{0}$ and $\sigma_{e}^{2}$ are random intercepts

2. $\beta_{1}$ is the slope of the average line: average increase across all regions in contraception usage for a unit change in Education $\left(X_{3}\right)$

Only two categories of education are significant. This means that contraception usage among different ages are different in different regions. Regional variation in the use of contraception among women with no education was seen to vary significantly higher than among other categories of education among women.

Table 9. Table for varying Education across regions :

\begin{tabular}{ll}
\hline & Model 1 \\
\hline (Intercept) & $-0.35(0.26)$ \\
Educationno educa & $-1.12^{* * *}(0.32)$ \\
Educationprimary & $-0.09(0.13)$ \\
Educationsecondar & $-0.35^{* *}(0.10)$ \\
Var:Region (Intercept) & 0.53 \\
Var: Region Educationno educa & 0.85 \\
Var: Region Educationprimary & 0.10 \\
Var: Region Educationsecondar & 0.04 \\
\hline
\end{tabular}

${ }^{* * *} p<0.001,{ }^{* *} p<0.01,{ }^{*} p<0.05$ 
71 Linda Vugutsa Luvai and Fred Ongango: Hierarchical Logistic Regression Model for Multilevel Analysis: An Application on Use of Contraceptives Among Women in Reproductive Age in Kenya

\subsubsection{Random Slopes Graphs}

The figures below show how the slopes vary across the regions.
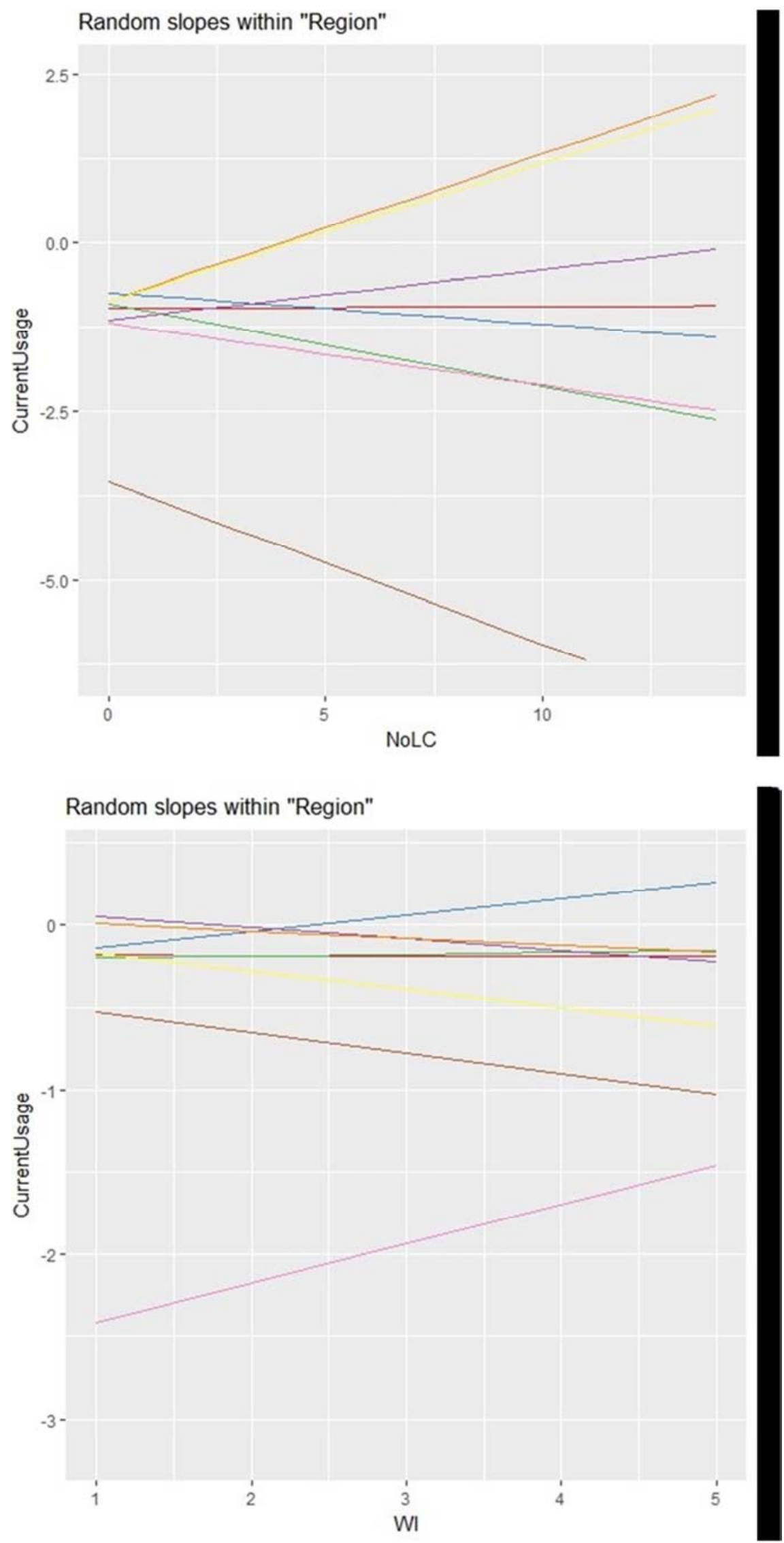


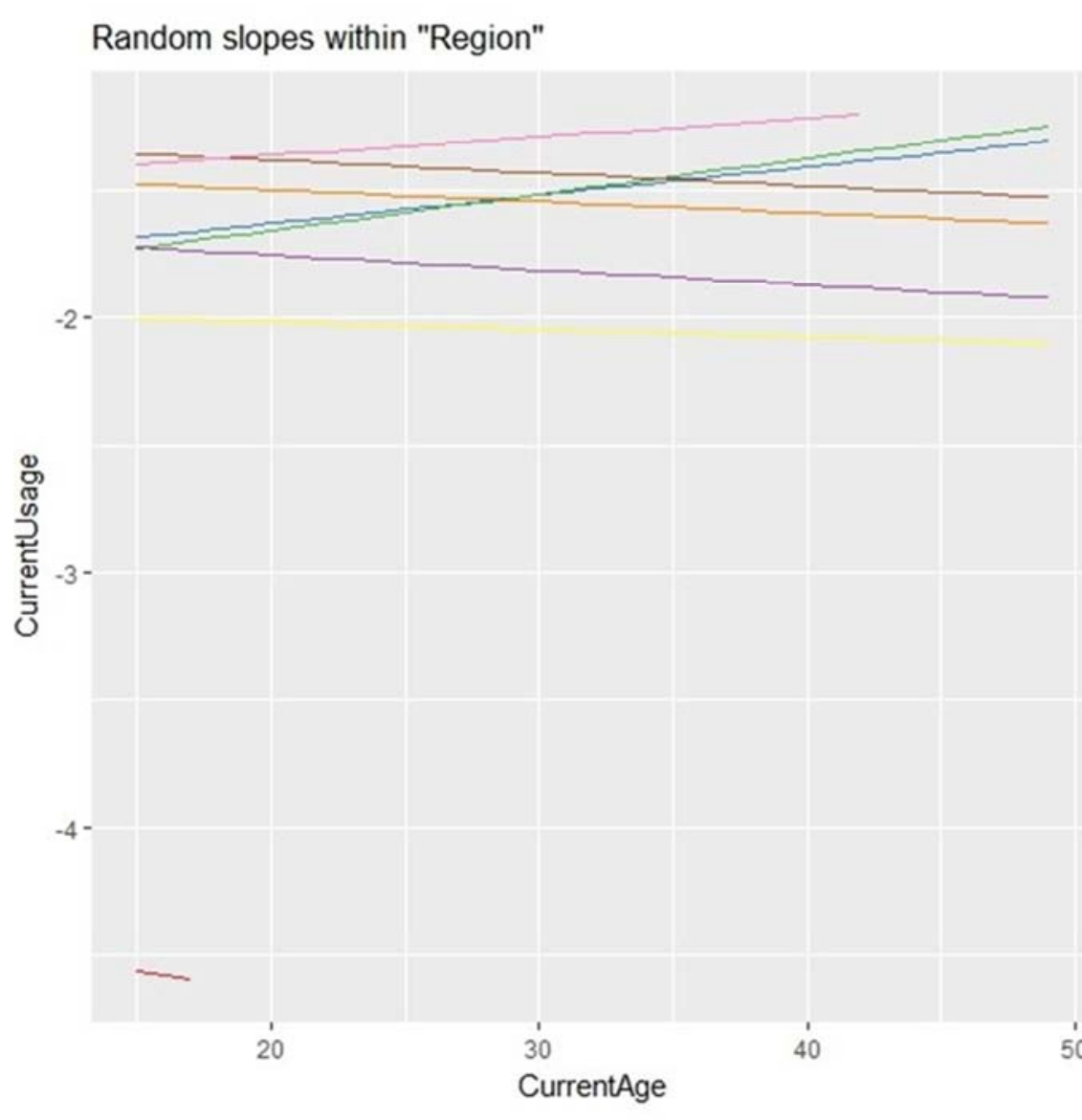

Random slopes within "Region"

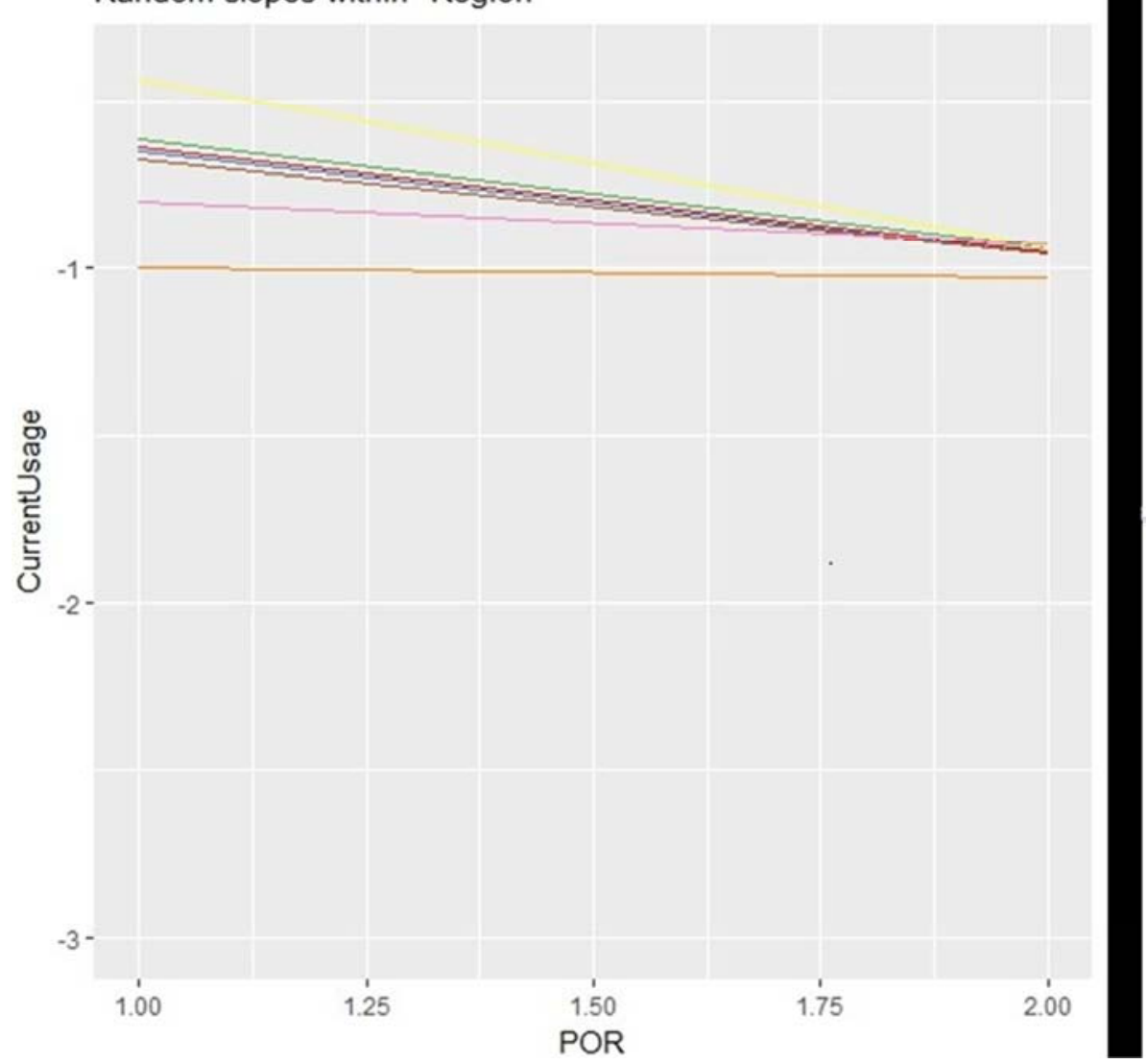


73 Linda Vugutsa Luvai and Fred Ongango: Hierarchical Logistic Regression Model for Multilevel Analysis: An Application on Use of Contraceptives Among Women in Reproductive Age in Kenya
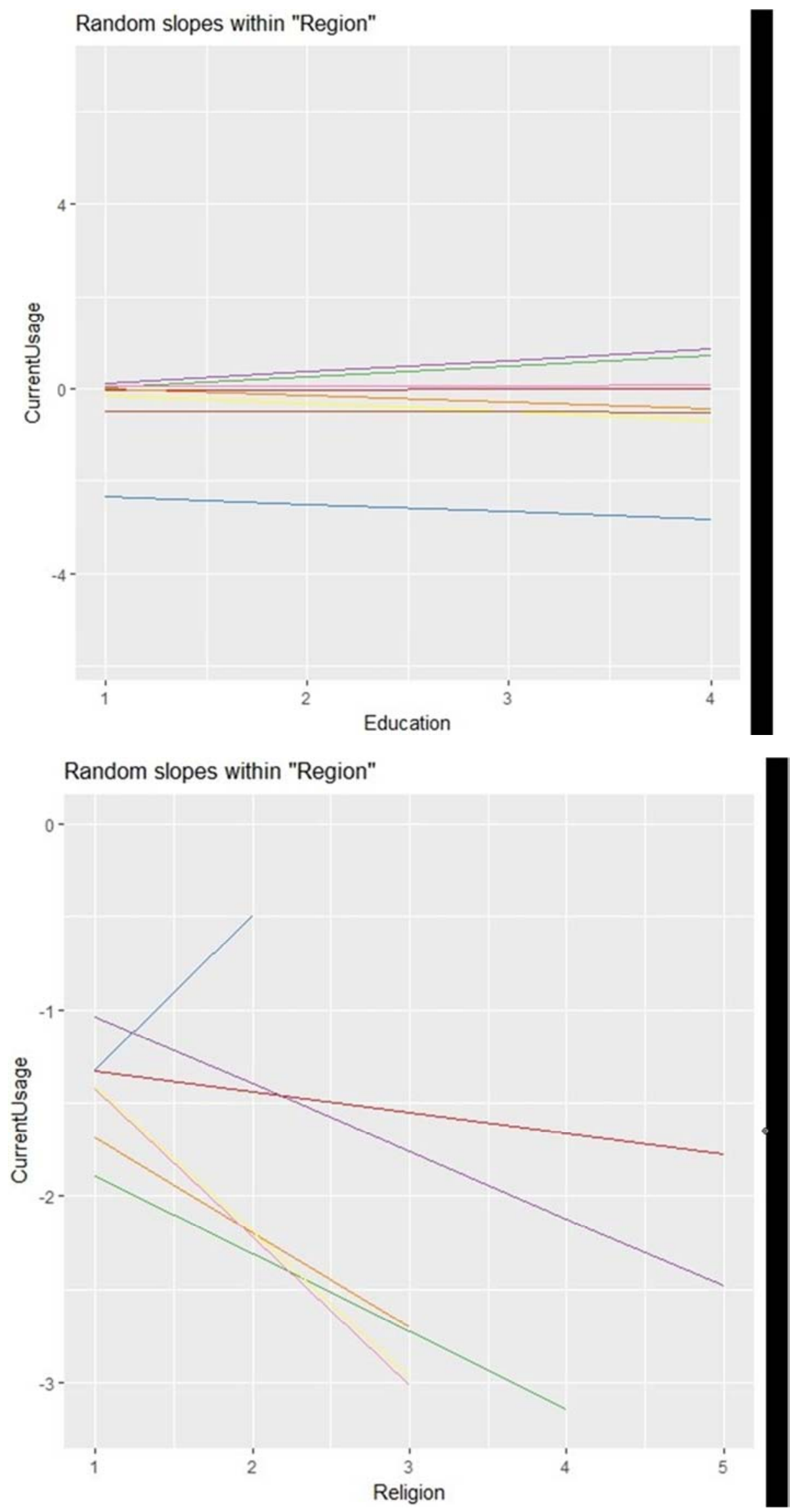

Figure 5. Random Slopes Graphs. 


\subsection{Multilevel Multivariate Logistic Modelling}

$$
\log \left\{\frac{\pi_{i j}}{1-\pi_{i j}}\right\}=\beta_{0 i j}+\beta_{1} X_{1}+\beta_{2 i j} X_{4 i j}+\beta_{3} X_{5}+\beta_{4} X_{7}+\beta_{5} X_{3}+\mu_{0 j}
$$

Where;

$$
\beta 0 i j=\beta 0+\mu 0 j+\mu 0 i j
$$

and

$$
\beta 2 i j=\beta 2+\mu 2 j+\mu 2 i j
$$

The multivariate model shows that the probability of using contraception is affected significantly with POR. While CurrentAge does not significantly influence contraception usage. When all other predictors are fixed in single-level multivariate analysis the probability of contraceptive usage is $18 \%$ higher in urban areas as compared to rural but for multilevel analysis the odds ratio is $33 \%$ higher in urban as compared to rural. To compare multilevel and single level analysis we compare their corresponding parameter estimates. From the last column of Table 4.9 it is seen that the coefficient under single-level analysis corresponding to POR covariate has been underestimated about $45.45 \%$ compared to multilevel estimates. In the analysis, wealth index (WI; only two categories are shown in Table 4.9) was found to be another important determinant to consider while predicting whether a woman will practice contraception. The wealth category that a woman belongs to will determine her contraception usage. The $\beta$ coefficient for NoLC from the single level model have been underestimated. On the other hand, the $\beta$ coefficient for Religion under standard logistic model has been greatly overestimated. Some notable overestimation or underestimation has happened for WI and Education explanatory variables. In the multivariate analysis framework variables, Religion (only one category as shown in Table 9 is not significant). This means that contraception usage depends on one's religion. Education, POR and NoLC have been found to be significantly associated with a woman's contraceptive usage. The multilevel analysis has also revealed that there exist variations in the mean effect of the predictors (except for CurrentAge) over the response variable CurrentUsage in Kenya. The variation is significant at $(\mathrm{p}<0.001)$.

\begin{tabular}{|c|c|c|c|}
\hline & Single Level & Multilevel Model & Over/Underestimation \\
\hline \multirow[t]{2}{*}{ (Intercept) } & $-1.83 * * *$ & $-1.76 * * *$ & $5.78 \%$ \\
\hline & $(0.09)$ & $(0.33)$ & \\
\hline \multirow[t]{2}{*}{ WIpoorer } & $-0.23 * * *$ & $-0.23 * * *$ & $0 \%$ \\
\hline & $(0.04)$ & $(0.04)$ & \\
\hline \multirow[t]{2}{*}{ WIpoorest } & $-0.86 * * *$ & $-0.85 * * *$ & $1.176 \%$ \\
\hline & $(0.05)$ & $(0.05)$ & \\
\hline \multirow[t]{2}{*}{$\underline{\text { WIricher }}$} & 0.07 & 0.06 & $16.67 \backslash \%$ \\
\hline & $(0.04)$ & $(0.04)$ & \\
\hline \multirow[t]{2}{*}{$\underline{\text { WIrichest }}$} & 0.06 & 0.03 & $100 \%$ \\
\hline & $(0.05)$ & $(0.05)$ & \\
\hline \multirow[t]{2}{*}{$\underline{\text { PORurban }}$} & $0.18^{* * *}$ & $0.33^{*}$ & $45.45 \%$ \\
\hline & $(0.03)$ & $(0.13)$ & \\
\hline \multirow[t]{2}{*}{$\underline{\text { CurrentAge }}$} & 0.00 & $-0.00 *$ & \\
\hline & $(0.00)$ & $(0.00)$ & \\
\hline \multirow[t]{2}{*}{ Educationno educa } & $-1.83 * * *$ & $-1.75^{* * *}$ & $4.57 \%$ \\
\hline & $(0.08)$ & $(0.08)$ & \\
\hline \multirow[t]{2}{*}{ Educationprimary } & $-0.21 * * *$ & $-0.28 * * *$ & $25 \%$ \\
\hline & $(0.05)$ & $(0.05)$ & \\
\hline \multirow[t]{2}{*}{ Educationsecondar } & $-0.26 * * *$ & $-0.30 * * *$ & $13.33 \%$ \\
\hline & $(0.05)$ & $(0.05)$ & \\
\hline \multirow[t]{2}{*}{$\underline{\text { Religionno relig }}$} & $0.77^{* * *}$ & $0.53 * * *$ & $45.28 \%$ \\
\hline & $(0.12)$ & $(0.13)$ & \\
\hline \multirow[t]{2}{*}{$\underline{\text { Religionother }}$} & 0.42 & 0.15 & $180 \%$ \\
\hline & $(0.32)$ & $(0.32)$ & \\
\hline \multirow[t]{2}{*}{$\underline{\text { Religionprotesta }}$} & $1.16^{* * *}$ & $0.96^{* * *}$ & $20.83 \%$ \\
\hline & $(0.05)$ & $(0.06)$ & \\
\hline \multirow[t]{2}{*}{ Religionroman ca } & $1.11^{* * *}$ & $0.88 * * *$ & $26.14 \%$ \\
\hline & $(0.06)$ & $(0.07)$ & \\
\hline \multirow[t]{2}{*}{$\underline{\text { NoLC }}$} & $0.30^{* * *}$ & $0.33 * * *$ & $9.09 \%$ \\
\hline & $(0.01)$ & $(0.01)$ & \\
\hline AIC & 35963.89 & 35570.05 & \\
\hline
\end{tabular}

Table 10. Parameters and standard errors of single level multivariate logistic model and multilevel multivariate model predicting the probability of contraceptive usage with random intercept Region (S.E.s are placed in parentheses). 


\begin{tabular}{llll}
\hline & Single Level & Multilevel Model & Over/Underestimation \\
\hline BIC & 36089.03 & 35720.23 \\
Log Likelihood & -17966.94 & -17767.03 \\
Deviance & 35933.89 & \\
Var: Region (Intercept) & & 0.79 \\
Var: Region PORurban & & 0.12 \\
Cov: Region (Intercept)PORurban & & -0.31 & \\
\hline
\end{tabular}

\subsection{Discussion}

Multilevel analyses using contraceptive binary data hasn't been done in Kenya. However, these analyses have found significant multilevel effects either at lower levels (individuals) or high level (regions). For instance, the study found that CurrentAge varies significantly across the regions and that there were strong regional effects on CurrentAge and the NoLC. Our analysis showed evidence $(p<0.001)$ of effects in higher level (Regions) in addition to higher significance in the lower level (individuals). Our study has continued to demonstrate the tendency for the single level logistic model to seriously bias the parameter estimates of observed covariates when analyzing multilevel data. However, the estimated bias generally differs depending on the estimation procedure used for the multilevel logistic model. This is consistent with the observation made by Goldstein and Rasbash (1996). The univariate analysis that we carried out showed that the predictor variable varied significantly across the regions at $(\mathrm{p}<0.001)$ while the multilevel multivariate analysis showed that the variables varied significantly with $(\mathrm{p}<0.001)$ apart from CurrentAge and POR which varied with $(\mathrm{p}<0.05)$. Consequently, our random slope modeling showed that their exists random effects at regional level of contraception usage among the women. We were able to see how contraception usage between different regions varied across the ages. Multilevel analysis has thus demonstrated that different regions have different random effects. For example, our analysis has demonstrated that NoLC that a woman has influences her contraception usage differently in different region. This are previously unrecognized effects.

\section{Conclusion and Recommendation(s)}

The 2014 KDHS on contraceptive binary data used multistage stratified cluster sampling. From our study, we found that for such hierarchical structured data, the multilevel effects are significant and have to be taken into consideration in logistic regression modeling, which leads to multilevel logistic regression modeling. Due to this, multilevel analysis enables the proper investigation of the effects of all explanatory variables measured at different levels (individual and regional level) on the response variable "currently using contraception", and finally the model gives appropriate estimates and conclusions about the parameters. A major reason for significant multilevel effects for such data might be dependencies between individual observations, due to sampling not being taken randomly but rather cluster sampling from geographical areas being used instead.

In conclusion we recommend that further work can be done to investigate more precisely the relationship between the extent of bias and factors such as level of within-regions correlation, level of within-county correlation and proportion of regions that has a single observation.

\section{Acknowledgements}

I would not have come to the complication of my masters program were it not for the Lord God granting me strength to work through day by day. I am therefore indebted to Him and give all thanks for His grace and mercy that have always been new every day. I would like to express my deepest gratitude to Professor Fred Onyango for his excellent guidance, sincere remarks and profitable engagement through this learning process and during the research period. I am glad that you were patient with me, you genuinely and patiently corrected by work and above all challenged me where necessary. I would also like to thank Dr. John Kekovole and Andrew Imbwaga (KNBS) who saw to it that I attain the data I required for this project. I am grateful to Maseno University for giving me an enabling environment to be able to study and particularly the Department of Statistics and Actuarial Science. Lastly, I want to appreciate my family, my dad Albert Luvai and mom Ruth Luvai who gave me unending and unparalleled support whenever I needed it. I am forever indebted to my parents for giving me opportunities and experiences that have made me who I am. 


\section{Appendix}

\section{Appendix A}

\section{Map of Kenya}

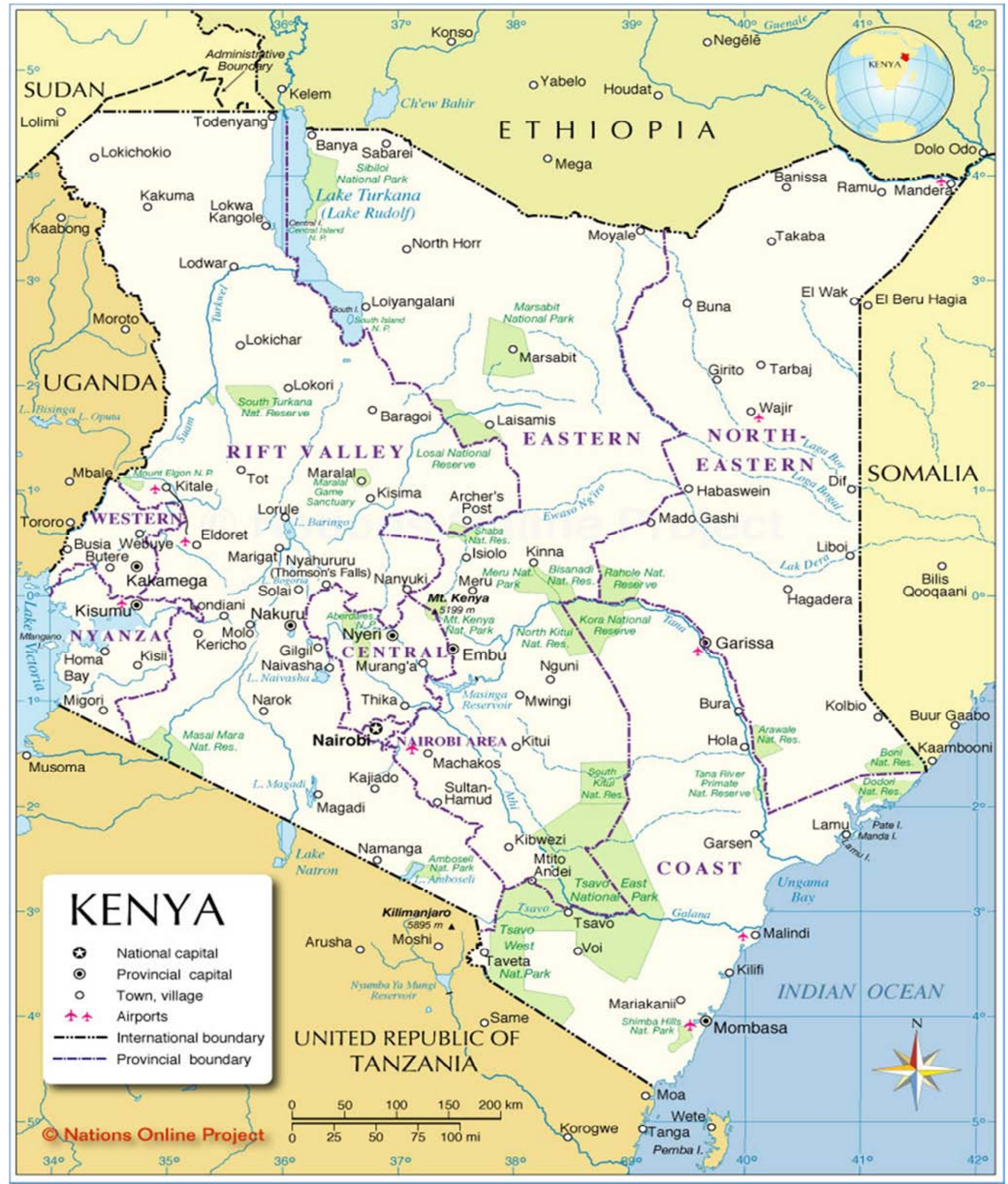

Figure 6. Map of Kenya. 


\section{Appendix B}

\section{Some R codes}

Here is the code for the null model.

null <-glmer (CurrentUsage $1 \quad+(1 \mid$ Region),

family=binomial

("logit"), data=MyData) null summary (null)

Random Intercept with explanatory variables.

model $2<$ - glmer (CurrentUsage $\sim$ CurrentAge $+(1 \mid$ Region), family=binomial ("logit"), data = MyData) model2 summary (model2) predprob <- fitted (model2) predlogit $<$ - logit (predprob) datapred <-unique (data. frame (cbind (predlogit=predlogit, Region=MyData\$Region, CurrentAge $=$ MyData $\$$ CurrentAge) $)$ ) xyplot (predlogit CurrentAge, data $=$ datapred, groups $=$ Region, type $=\mathrm{c}(" \mathrm{p} "$, "l", "g"), col = "blue", xlim = c $(9,51)$, ylim = c $(-4,4))$

Random effects of intercept graph sjp. lmer $($ model 2 , facet. grid $=$ FALSE, sort. est $=$ "sort. all", y. offset $=.4$ )

Getting Conditional Variances

Attr (ranef (model2, postVar=T) [[1]], "postVar") u0<-ranef (model2, condVar=TRUE) uose $<$-sqrt (attr (u0 [[1]],"postVar") $[1,$,$] ) uose str (u0 [[1]]) regions <$-as. factor (rownames (u0 $[[1]]))$ regions $\mathrm{u} 0 \mathrm{tab}<-$-cbind $\quad$ ("regions"=regions, $\mathrm{u} 0 "=\mathrm{u} 0$ [[1]],"uose"= uose)colnames (u0tab) [2]<-"u0"u0tab<-u0tab [order (u0tab\$u0),] u0tab<-cbind (u0tab, c (1:dim (u0tab) [1])) u0tab $<-$ u0tab [order (u0tab\$regions), colnames (u0tab) [4]<"u0regions" plot (u0tab\$u0regions, u0tab\$u0, type="n", $\mathrm{xlab}=$ "u_region", ylab = "conditional modes of r.e. for Region", ylim $=\mathrm{c}(-4,4))$ segments $(\mathrm{u} 0$ tab\$u0regions, u0tab\$u0$1.96 * \mathrm{u} 0$ tab\$ uose, u0tab\$u0regions, u0tab\$u0+ $1.96 * \mathrm{u} 0$ tab\$uose)points (u0tab\$u0regions, u0tab\$u0, col= "blue") abline ( $\mathrm{h}=0, \mathrm{col}=$ "red")

Univariate analysis codes

model4<-glmer

(CurrentUsage $\sim$ AgeG + POR + Religion + WI + NoLC +

Education +(1|MyData\$Region), family=binomial ("logit"), data=MyData, verbose=FALSE) model4 summary (model4) texreg (model4, model4b) model4b $<$-glm (CurrentUsage $\sim$ AgeG $+\mathrm{POR}+$ Religion $+\mathrm{WI}+\mathrm{NoLC}+$

Education, family=binomial ("logit"), data=MyData) model4b summary (model4b) xtable (anova (model4, model4b)) texreg (list (model4b, model4))

model5<-glmer

(CurrentUsage $\sim \mathrm{WI}+\mathrm{POR}+$ CurrentAge + Education + Religion

$+\quad$ NoLC $+(1 \mid$ Region $), \quad$ family=binomial ("logit"), control=glmerControl (optimizer="bobyqa"), $\mathrm{nAGQ}=10$, data $=$ MyData) model5 summary (model5)

Random slope models $\mathrm{R}$ codes

model7<-lmer (CurrentUsage $\sim \mathrm{WI}+(\mathrm{WI} \mid$ Region), family=binomial ("logit"), data=MyData) model7 summary (model7) VarCorr (model7) plot (ranef (model7) [, 1], ranef (model7) [, 8], xlab="intercepts

(u_i)", ylab="slopes (v_i)") $\quad$ model $7 b<-$-lmer (CurrentUsage $\sim \mathrm{WI} * \mathrm{POR}+(\mathrm{WI} \mid$ Region), family=binomial ("logit"), data=MyData) model7b summary (model7b) texreg (list (model7, model7b))

model8<-lmer (CurrentUsage $\sim \mathrm{NoLC}+(\mathrm{NoLC} \mid$ Region), family=binomial ("logit"), data=MyData) model8 xtable (model8) summary (model8) texreg (model8)

mode19<-lmer (CurrentUsage $\sim \mathrm{POR}+(\mathrm{POR} \mid$ Region $)$, family=binomial ("logit"), data=MyData) model9 summary (model9)

model10a<-glmer (CurrentUsage $\sim$ CurrentAge $+\mathrm{I}$ $\left(\right.$ CurrentAge $\left.{ }^{\wedge}\right)+\quad(1 \mid$ Region$), \quad$ family=binomial ("logit"), data=MyData) model10a summary (model10a)

model10<-glmer (CurrentUsage $\sim$ CurrentAge+I

$\left(\right.$ CurrentAge $\left.{ }^{\wedge} 2\right)+\quad$ (CurrentAge|Region), family=binomial ("logit"), data=MyData) model10 summary (model10) anova (model10a, model10) anova (model10, Model13)

plot (model10)

xyplot (fitted (model10) CurrentAge, groups $=$ NoLC, col=tim.colors (length (unique $($ MyData\$NoLC)) ), lwd=15, $\mathrm{pch}=1$, data $=$ MyData, $x \lim =$ MyData $\$$ NoLC, ylim $=\mathrm{c} \quad(15$, 50)) xyplot (fitted (model10) CurrentAge|AgeG, groups $=$ Region, lwd=1, $\mathrm{t}=" \mathrm{~b} ", \mathrm{pch}=1$, data $=$ MyData, ylim $=\mathrm{c}$ $(15,50))$

model11<-glmer

(CurrentUsage $\sim$ Education+(Education|Region),

family=binomial ("logit"), data=MyData) model11 summary (model11) model12<-glmer

(CurrentUsage $\sim$ Religion + (Religion $\mid$ Region), family=binomial ("logit"), data=MyData) model12 summary (model12)

Random slope graphs $\mathrm{R}$ code

ranef (model8) fixef (model8) ranef (model9) fixef (model9) ranef (model10) fixef (model10) ranef (model11) fixef (model11)

sjp.lmer (model7, type="rs.ri", vars="WIrichest", sample.n =8) sjp.lmer (model8, type="rs.ri", vars="NoLC", sample.n =8) sjp.lmer (mode19, type="rs.ri", vars="POR", sample.n =8) sjp.lmer (model10, type="rs.ri", vars="CurrentAge", sample.n $=8$ ) sjp.lmer (model11, type="rs.ri", vars="Education", sample.n =8) sjp.lmer (model12, type="rs.ri", vars="Religion", sample.n $=8$ )

Checking interactions

Model13<-glmer (CurrentUsage $\sim$ CurrentAge*NoLC+ I (CurrentAge $\left.{ }^{\wedge} 2\right)+\mathrm{POR}+(\mathrm{POR} \mid$ Region $)$,

Model13 summary (Model13)

Multilevel multivariate analysis

model14<-glmer

(CurrentUsage $\sim \mathrm{WI}+\mathrm{POR}+$ CurrentAge + Education +

Religion+NoLC+(POR|Region), family=binomiall ("logit"), data=MyData) model14 summary (model14) anova (model14, model5)

\section{References}

[1] Achana, F. S., Bawah, A. A., Jackson, E. F., Welaga, P., Awine, T., AsuoMante, E., Oduro, A., Awoonor-Williams, J. K., and Phillips, J. F. (2015). Spatial and socio-demographic determinants of contraceptive use in the upper east region of ghana. Reproductive health, 12(1):29. 
[2] Bongaarts, J. (2011). Can family planning programs reduce high desired family size in sub-saharan africa? International Perspectives on Sexual and Reproductive Health, 37(4):209216.

[3] Christensen, R. (2006). Log-linear models and logistic regression. Springer Science \& Business Media.

[4] Darko, J. A. (2016). Reproductive and child health: contraceptive knowledge, use and factors affecting contraceptive use among female adolescents (15-19 years) in Ghana. PhD thesis, KWAME NKRUMAH UNIVERSITY OF SCIENCE AND TECHNOLOGY.

[5] Ettarh, R. R. and Kyobutungi, C. (2012). Physical access to health facilities and contraceptive use in kenya: Evidence from the 2008-2009 kenya demographic and health survey. African journal of reproductive health, 16(3).

[6] Gelman, A. and Hill, J. (2006). Data analysis using regression and multilevel/hierarchical models. Cambridge university press.

[7] Gilmour, A. R., Thompson, R., and Cullis, B. R. (1995). Average information reml: an efficient algorithm for variance parameter estimation in linear mixed models. Biometrics, pages 1440-1450.

[8] Hair, J. F., Anderson, R. E., Babin, B. J., and Black, W. C. (2010). Multivariate data analysis: A global perspective, volume 7. Pearson Upper Saddle River, NJ.

[9] Khan, H. R. and Shaw, E. (2011). Multilevel logistic regression analysis applied to binary contraceptive prevalence data.

[10] Levandowski, B. A., Kalilani-Phiri, L., Kachale, F., Awah, P., Kangaude, G., and Mhango, C. (2012). Investigating social consequences of unwanted pregnancy and unsafe abortion in malawi: the role of stigma. International Journal of Gynecology \& Obstetrics, 118(S2).

[11] Makau, A., Waititu, A. G., and Mungï¿œatu, J. K. (2016). Multinomial logistic regression for modeling contraceptive use among women of reproductive age in kenya. American Journal of Theoretical and Applied Statistics, 5(4):242-251.

[12] Malhotra, A., Schuler, S. R., et al. (2005). Womenï ¿œs empowerment as a variable in international development. Measuring empowerment: Crossdisciplinary perspectives, pages $71-88$.

[13] Manlove, J., Welti, K., Barry, M., Peterson, K., Schelar, E., and Wildsmith, E. (2011). Relationship characteristics and contraceptive use among young adults. Perspectives on Sexual and Reproductive Health, 43(2):119-128.
[14] Menard, S. (2002). Applied logistic regression analysis. Number 106. Sage.

[15] Mohamed, S. F., Izugbara, C., Moore, A. M., Mutua, M., Kimani-Murage, E. W., Ziraba, A. K., Bankole, A., Singh, S. D., and Egesa, C. (2015). The estimated incidence of induced abortion in kenya: a cross-sectional study. BMC pregnancy and childbirth, 15(1):185.

[16] Nsubuga, H., Sekandi, J. N., Sempeera, H., and Makumbi, F. E. (2016). Contraceptive use, knowledge, attitude, perceptions and sexual behavior among female university students in uganda: a cross-sectional survey. BMC women's health, 16(1):6

[17] Ojakaa, D. (2008a). The fertility transition in kenya: patterns and determinants.

[18] Ojakaa, D. (2008b). Trends and determinants of unmet need for family planning in kenya.

[19] Oluwaseun, O. J., Babalola, B. I., and Gbemisola, A. Determinants of contraceptive use among female adolesents in nigeria.

[20] ON, A., OF, U., and AMONG, C. (2017). Multilevel logistic regression model: An.

[21] Sonfield, A., Hasstedt, K., Kavanaugh, M. L., and Anderson, R. (2013). The social and economic benefits of womenï ¿œs ability to determine whether and when to have children.

[22] Standard Reporter, K. (2015). 30 years on, myths and misconceptions still a barrier to contraceptive use. Read more at:

https://www.standardmedia.co.ke/health/article/2000163756/3 0years-on-myths-and-misconceptions-still-a-barrier-tocontraceptive-use.

[23] Tumlinson, K., Pence, B. W., Curtis, S. L., Marshall, S. W., and Speizer, I. S. (2015). Quality of care and contraceptive use in urban kenya. International perspectives on sexual and reproductive health, 41(2):69. Vaughn, B. K. (2008). Data analysis using regression and multilevel/hierarchical models, by gelman, a., \& hill, j. Journal of Educational Measurement, 45(1):94-97.

[24] Worku, A. G., Tessema, G. A., and Zeleke, A. A. (2015). Trends of modern contraceptive use among young married women based on the 2000, 2005, and 2011 ethiopian demographic and health surveys: a multivariate decomposition analysis. PloSone, 10(1):e0116525. 